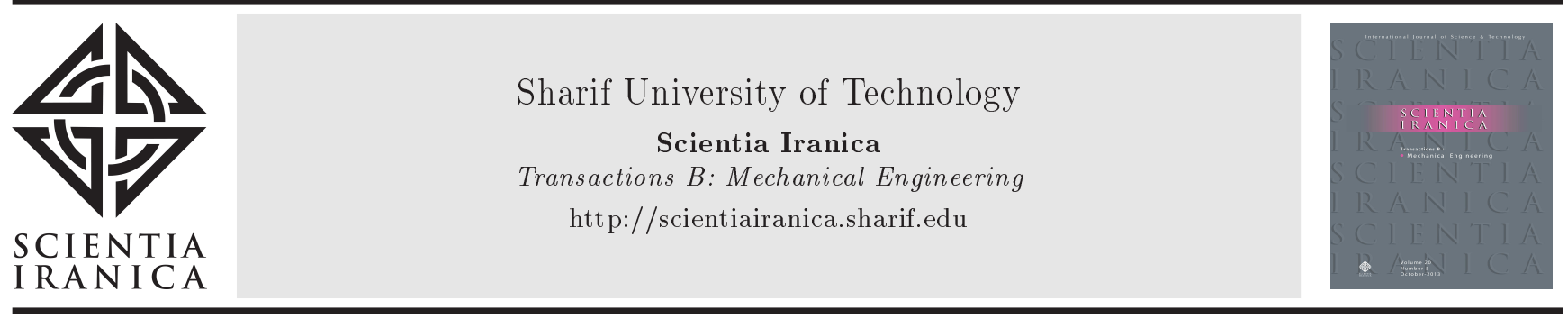

\title{
A modified wavelet energy rate-based damage identification method for steel bridges
}

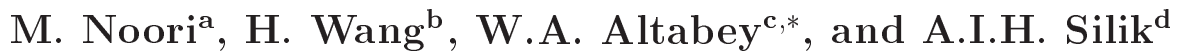 \\ a. International Institute for Urban Systems Engineering, Southeast University, Nanjing 210096, China; and Mechanical \\ Engineering and ASME Fellow, California Polytechnic State University, San Luis Obispo, California, USA. \\ b. State University of New York, Buffalo, NY 14260, USA. \\ c. International Institute for Urban Systems Engineering, Southeast University, Nanjing 210096, China, Nanjing Zhixing \\ Information Technology Company Nanjing; China; and Department of Mechanical Engineering, Faculty of Engineering, \\ Alexandria University, Alexandria 21544, Egypt. \\ d. International Institute for Urban Systems Engineering, Southeast University, Nanjing 210096, China.
}

Received 12 March 2018; accepted 2 July 2018

\section{KEYWORDS \\ FBG strain sensor; \\ Wavelet packet \\ transform; \\ Damage identification; \\ Modified wavelet \\ packet energy rate.}

\begin{abstract}
Strain is sensitive to damage, especially in steel structures. However, a traditional strain gauge does not fit bridge damage identification because it only provides the strain information of the point, where it is set up. While traditional strain gauges suffer from drawbacks, a long-gage FBG strain sensor is capable of providing the strain information of a certain range, in which all the damage information within the sensing range can be reflected by the strain information provided by FBG sensors. The wavelet transform is a new way to analyze the signals, capable of providing multiple levels of details and approximations of the signal. In this paper, a wavelet packet transform-based damage identification is proposed to identify the steel bridge damage numerically and with experimentally to validate the proposed method. The strain data obtained via long-gage FBG strain sensors are transformed into a modified wavelet packet energy rate index first to identify the location and severity of damage. The results of numerical simulations show that the proposed damage index is a good candidate that is capable of identifying both the location and severity of damage under noise effect.
\end{abstract}

(C) 2018 Sharif University of Technology. All rights reserved.

\section{Introduction}

Since steel bridges suffer from complex working conditions during their service life, heavy working loads and other effects damage structures and accelerate the development of damages. With the accumulation of damages to structures, the safety of both the structure and human life can be threatened and may directly

*. Corresponding author. Tel.: +86-17368476644 E-mail addresses: mnoori@outlook.com (M. Noori); Hwang48@buffalo.edu (H.Wang); Wael.altabey@gmail.com (W.A. Altabey); silikth@gmail.com (A.I.H. Silik)

doi: $10.24200 /$ sci.2018.20736 result in tragedies of life and property $[1,2]$. Therefore, structural damage identification has become an important research topic in the engineering protection field [3]. Over the last two decades, the considerable development of integrated monitoring systems for new and existing structures worldwide, such as steel bridges, has been recognized as an important tool to solve structural damage identification problem. It is commonly stated in the structural health monitoring literature that damage identification approaches are classified as methods based on time domain analysis [4], methods based on modal parameters [5], and methods based on time-frequency domain analysis [6]. The majority of these techniques are based on vibration that require data acquisition instruments to be fixed 
on the bridge directly, which may be difficult and timeconsuming; however, it is effective in giving a warning to people if there is any indication of hazard on Bridge's condition. These methods become a more important part of bridge monitoring systems.

In recent years, there have been movement towards the development of indirect vibration methods based on a vehicle response moving over a bridge. These methods aimed to reduce the need of installing instruments directly on bridge to achieve more efficiency and low cost. In related development, Bu et al. [7] and McGetrick \& Kim [8] investigated numerically a bridge condition using the dynamic response of a vehicle running along a beam to evaluate related damage based on reduction of stiffness. The results showed that vehicle speed, road surface roughness, measurement noise, and numerical model errors did not have significant effect on the accuracy of the method. In addition, González et al. [9] innovated a novel algorithm using the vehicle acceleration as an algorithm input to determine the damping of a bridge. It was shown that the algorithm could be used for bridge stiffness identification. It was also stated that the algorithm was not highly sensitive to signal with lowlevel noise, the road roughness, and model errors.

Furthermore, current damage detection techniques can be classified into two broad categories: local and global damage identifications. Most global damage identification methods rely on damage-induced changes in the dynamic properties of the identified structure. In these identification methods, system parameters, such as frequencies, deflected mode shapes, strain energy, flexibility matrix, etc., are used as part of the damage index [10]. Among the discussed damage detection methods, some are directly based on Fourier transform (e.g., natural frequency-based damage identification methods). Fourier transform provides information in the frequency domain and is not capable of detecting when (or where) a particular damage occurs. To overcome this disadvantage, Dennis Gabor proposed the Short-Time Fourier Transform (STFT). This wellknown windowing technique divides the signals into a series of sections. Each time, STFT is done within a small window that represents a section of the signal. Thus, information on both frequency and time domains can be kept. However, STFT has a disadvantage: the information about time and frequency (or space and frequency) is acquired with limited precision. According to the Heisenberg uncertainty theory, this limitation is determined by the size of the timefrequency window. Once the window size is set, it is the same for all frequencies; therefore, a higher resolution in time and frequency domains (or space and frequency domain) cannot be acquired simultaneously.

The Wavelet Transform (WT) is a relatively new signal-processing tool to analyze data. Based on the theory of WT, it can be viewed as an extension of traditional STFT transform with adjustable window location and size. With its unique merit of examining signals with a "zoom lens having an adjustable focus", the original signal is separated into two different levels of details and approximations [11-13]. Therefore, transient information of the signal can be retained. The wavelet transform as a technique for structural damage identification has been introduced recently due to its capability of capturing the transient behavior of a signal and analyzing it in time and frequency domains, as investigated by several researchers. Hester et al. [14] demonstrated the ability of the wavelet transform to get information from time-frequency domains, while Zhao et al. [15-17] employed the structural mode shapes that extracted from the finite element model of a simply supported reinforced concrete beam for damage identification using different types of wavelets. Reda Taha et al. [18] discussed in detail various aspects related to wavelet transform as a technique for structural damage. Nguyen and Tran [19] provided a method based on Symlet wavelet to evaluate bridge cracks from vehicle displacement response. It was concluded that cracks can be detected; however, higher speeds give poor detection than low speed. Lee et al. [20] provided an algorithm for truss bridge based on the continuous relative wavelet entropy. It was concluded that damage could be detected; however, computation cost is very large for the real-life monitoring. Although significant research has been carried out in the area of structural health monitoring to make structures work safely, highly reliable and practical damage identification methods are still lacking and many disasters occur due to lack of damage identification.

Strain measurements have proved to be sensitive to damage. However, if traditional strain gauges are not installed exactly on the damaged locations, the damage detection result will not be accurate. Thus, the idea of distributed sensing system was proposed to overcome the limitation of the traditional strain gauge in obtaining the strain, where they are installed [21]. Distributed sensing system is different from multipoint sensing system. It is capable of capturing the information within a certain range of the structure, leading to integrated information of the entire structure.

However, this requirement of distributed sensing systems was unreachable until Horiguchi et al. [22] proposed the relationship between the strain and Brillouin frequency shift in optical fibers. Based on this relationship, a concept of distributed sensing system was proposed. Subsequently, Fiber Bragg Grating (FBG) sensors [23], Brillouin Optical Time Domain Reflectometer (BOTDR) sensors [24,25], and Brillouin Optical Time Domain Analysis (BOTDA) have been widely used in long-gage strain monitoring systems.

FBG sensors are based on the principle that the 
wavelength of the reflective signal from the grating changes when it has longitudinal deformation. By measuring the changes of wavelength, accurate deformation of the grating can be achieved. Based on wavelengthdivision multiplexing technology, several FBG sensors can be combined in one fiber to achieve multipoint sensing. For long-gage FBG sensors [26], fiber Bragg gratings are packaged in series to extend the effective sensing length. FBG sensors have their own merit: the signals used in FBG sensors are wavelength-modulated signals, meaning that they do not need to suffer from the limitations of inaccurate phase measurement used in other kinds of fiber optic sensors.

BOTDR and BOTDA sensors are based on Brillouin scattering [24,25]. Once there is a change in strain or temperature on the fiber, the central frequency of Brillouin scattering light changes. Based on the relationship, Brillouin scattering light can be used to acquire changes in strain and temperature. For BOTDR and BOTDA sensors, any part of the fiber is both a sensing unit and a signal transmission unit, leading to their capability of spatial continuous measurement.

BOTDR/BOTDA sensors and FBG sensors have different merits based on their sensing concept. FBG sensors have better accuracy in both dynamic and static sensing. However, theoretically, FBG sensors are point sensors. Their sensing length is limited. The accuracy and sampling rate of BOTDR/BOTDA sensors is lower than those of FBG sensors. However, they are more suitable for distributed sensing, especially in large-scale structures. With the consideration of the small scale of the laboratory, long-gage FBG sensors are adopted in this research.

In this paper, the goal is to investigate the effectiveness of a modified wavelet energy rate-based damage identification method for steel bridges elements (beam and frame). Both simulated and experimental experiments are utilized to validate the proposed method. Measured dynamic signals from structures are first decomposed into the wavelet packet components. Then, the modified wavelet packet strain energy rate index is calculated based on the wavelet packet components and is, then, used to locate the damage and assess the severity of damage. Different scenarios are considered to validate the proposed damage identification method. The simulated test results show that the proposed damage identification method is able to detect both location and severity of damages to a structure.

\section{Theoretical background}

\subsection{Wavelet and continuous wavelet transform}

Wavelet analysis is a signal processing method that relies on the introduction of an appropriate basis and characterization of the signal by the distribution of amplitude on the basis [27]. If the wavelet is required to form a proper orthogonal basis, it has the advantage that an arbitrary function can be uniquely decomposed, and the decomposition can be inverted. The wavelet is a smooth and quickly vanishing oscillating function with good localization in both frequency and time. A wavelet family, $\psi_{a, b}(t)$, is a set of elementary functions generated by dilations and translations of a unique admissible mother wavelet, $\psi(t)$ :

$$
\psi_{a, b}(t)=\frac{1}{\sqrt{a}} \psi\left(\frac{t-b}{a}\right),
$$

where $b \in R, a>0$ are the scale and translation parameters, respectively, and $t$ is time (or location, if wavelet transform is utilized in spatial distributed signal). As scale parameter $a$ increases, the wavelet becomes wider. Therefore, each parameter shows the signal at different scales and with variable time (or space localization).

The wavelet transform (in its continuous or discrete version) correlates function $f(t)$ with $\psi_{a, b}(t)$. The Continuous Wavelet Transform (CWT) is the sum of all time of the signal multiplied by a scaled and shifted version of a mother wavelet:

$$
C(a, b)=\frac{1}{\sqrt{a}} \int_{-\infty}^{\infty} f(t) \psi\left(\frac{t-b}{a}\right) d t .
$$

The results of the transform are wavelet coefficients, determining how the wavelet function signal expresses the signal. Hence, sharp transitions $f(t)$ create wavelet coefficients with large amplitudes, and this precisely is the basis of the proposed damage identification method.

The CWT has an inverse: The inverse CWT helps to recover the signal from its coefficients $C(a, b)$ and is defined as follows:

$$
f(t)=\frac{1}{K_{\psi}} \int_{a}^{+\infty} \int_{-\infty}^{+\infty} C(a, b) \psi_{a, b}\left(\frac{t-b}{a}\right) \frac{1}{a^{2}} d a d t,
$$

where constant $K_{\psi}$ is:

$$
K_{\psi}=\int_{0}^{+\infty} \frac{|\hat{\psi}(\omega)|^{2}}{\lfloor\omega\rfloor} d \omega
$$

\subsection{Discrete Wavelet Transform (DWT) and Wavelet Packet Transform (WPT)}

There is still a drawback to the CWT: A very large number of wavelet coefficients $C(a, b)$ are generated during the analysis [28]. It can be shown that the CWT is highly redundant, because it is not necessary 
to use the full domain of $C(a, b)$ to reconstruct $f(t)$. Therefore, instead of using a continuum of dilations and translations, discrete values of the parameters are used in Discrete Wavelet Transform (DWT). The dilation is defined as $a=2^{j}$, and the translation parameter takes the values of $b=k 2^{j},(j, k) \in Z$, and $Z$ is a set of integers. It has been proved that DWT can fully decompose a signal without losing any part of it. This sampling of the coordinates is referred to as dyadic sampling, because consecutive values of the discrete scales differ by a factor of 2 [29]. Using the discrete scales, one can define the DWT:

$$
\begin{aligned}
C_{j, k} & =2^{-\frac{j}{2}} \int_{-\infty}^{+\infty} f(x) \psi\left(2^{-j} x-k\right) d x \\
& =\int_{-\infty}^{+\infty} f(x) \psi_{j, k}(x) d x .
\end{aligned}
$$

The signal resolution is defined as the inverse of the scale $1 / a=2^{-j}$, and integer $j$ is referred to as the level. As the decomposition level increases, the frequency resolution increases, and the smaller bandwidth components of the signal can be obtained.

The signal can be reconstructed from wavelet coefficients, $C_{j, k}$, and the reconstruction algorithm is called the Inverse Discrete Wavelet Transform (IDWT):

$$
f(x)=\sum_{j=-\infty}^{\infty} \sum_{k=-\infty}^{\infty} C_{j, k} 2^{-j / 2} \psi\left(2^{-j} x-k\right) .
$$

One possible drawback to the DWT is that the frequency resolution is quite poor in the high-frequency region. It is difficult for DWT to discriminate signals containing close high-frequency components. The Wavelet Packet Transform (WPT) is one extension of the DWT that provides complete level-by-level decomposition. The wavelet packets are alternative bases formed by linear combinations of the usual wavelet functions. The decomposed subcomponents can be treated as narrow-band signals under high decomposition levels.
Wavelet packets consist of a set of linearly combined usual wavelet functions. The wavelet packets inherit properties, such as orthonormality and time-frequency localization, from their corresponding wavelet functions [30]. A wavelet packet is a function with three indices, $\psi_{j, k}^{i}(t)$ where integers $i, j$ and $k$ are the modulation, the scale, and the translation parameter, respectively.

$$
\psi_{j, k}^{i}(t)=2^{j / 2} \psi^{i}\left(2^{j} t-k\right), \quad i=1,2, \ldots
$$

The wavelets, $\psi^{i}$, are obtained from the following recursive relationships:

$$
\begin{aligned}
& \psi^{2 i}(t)=\sqrt{2} \sum_{k=-\infty}^{\infty} h(k) \psi^{i}(2 t-k), \\
& \psi^{2 i+1}(t)=\sqrt{2} \sum_{k=-\infty}^{\infty} g(k) \psi^{i}(2 t-k) .
\end{aligned}
$$

The first wavelet is the so-called mother wavelet function:

$$
\psi^{1}(t)=\psi(t)
$$

Discrete filters $h(k)$ and $g(k)$ are quadrature mirror filters associated with the scaling function and the mother wavelet function, which can be treated as low-pass and high-pass filters. A quadrature mirror filter is a filter whose magnitude response is the mirror image around $\pi / 2$ of that of another filter. There are quite a few mother wavelets reported in the literature. Most of these mother wavelets are developed to satisfy some important properties, such as the invertibility and the orthogonality. Daubechies developed a family of mother wavelets based on the solution of a dilation equation. One of these wavelets, DB20, is adopted in this study.

Similar to the FT, any measurable and squareintegrable function can be decomposed into wavelet packets. The decomposition process is a recursive filterdecimation operation. Figure 1 shows a full WPT tree

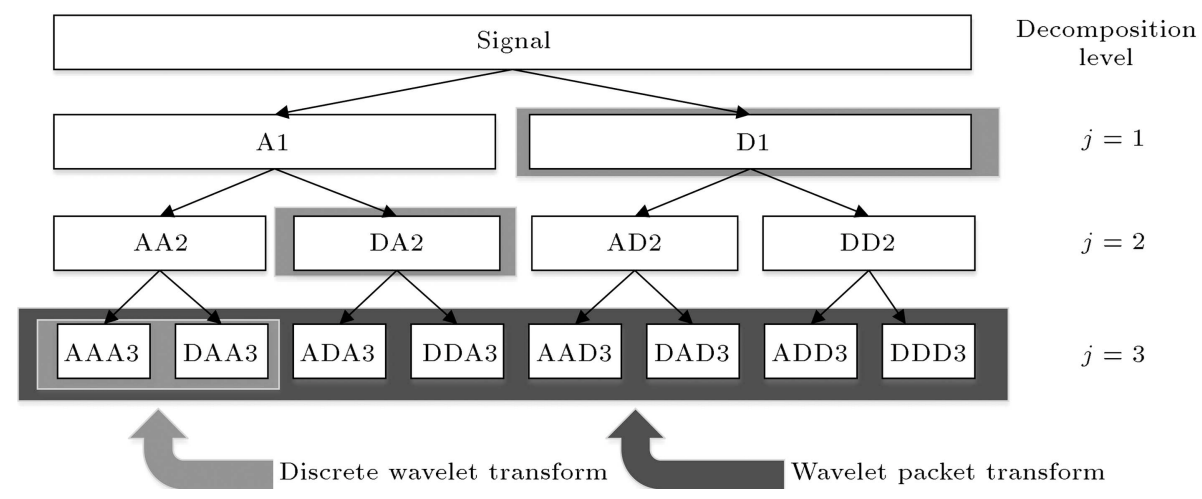

Figure 1. Three-level wavelet transform and packet transform. 
of a time-domain signal, $f(t)$, up to the 3rd level of decomposition. Figure 1 shows that the DWT consists of one high-frequency term from each level and one lowfrequency residual from the last level of decomposition. The WPT, on the other hand, contains complete decomposition at every level and, hence, can achieve a higher resolution in the high-frequency region. The recursive relations between the $j$ th and the $j+1$ th level components are:

$$
\begin{aligned}
& f_{j}^{i}\left(t=f_{(j+1)}^{(2 i-1)}(t)+f_{(j+1)}^{2 i}(t),\right. \\
& f_{j+1}^{2 i-1}(t)=H f_{j}^{i}(t), \\
& f_{j+1}^{2 i}(t)=G f_{j}^{i}(t),
\end{aligned}
$$

where $H$ and $G$ are filtering-decimation operators and are related to discrete filters, $h(k)$ and $g(k)$, through:

$$
\begin{aligned}
& H(t)=\sum_{k=-\infty}^{\infty} h(k-2 t), \\
& G(t)=\sum_{k=-\infty}^{\infty} g(k-2 t) .
\end{aligned}
$$

After $j$ levels of decomposition, original signal $f(t)$ can be expressed as follows:

$$
f(t)=\sum_{i=1}^{2^{j}} f_{j}^{i}(t)
$$

The wavelet packet component signal, $f_{j}^{i}(t)$, can be expressed by a linear combination of wavelet packet functions, $\psi_{j, k}^{i}(t)$, as follows:

$$
f_{j}^{i}(t)=\sum_{k=-\infty}^{\infty} c_{j, k}^{i} \psi_{j, k}^{i}(t)
$$

The wavelet packet coefficients, $c_{j, k}^{i}$, can be obtained from:

$$
c_{j, k}^{i}=\int_{-\infty}^{+\infty} f(t) \psi_{j, k}^{i}(t) d t
$$

Providing that the wavelet packet functions are orthogonal:

$$
\psi_{j, k}^{m}(t) \psi_{j, k}^{n}(t)=0, \quad \text { if } m \neq n \text {. }
$$

Each component in the WPT tree can be viewed as the output of a filter tuned to a particular basis function; thus, the whole tree can be regarded as a filter bank. At the top of the WPT tree (lower level), the WPT yields good resolution in the time domain, yet poor resolution in the frequency domain. At the bottom of the WPT tree (higher level), the WPT results in good resolution in the frequency domain, yet poor resolution in the time domain.

\subsection{Modified wavelet packet energy rate}

\subsubsection{The Continuous Wavelet Transform (CWT)} wavelet packet energy

Energy concentration is one of the reliable features of time-frequency data processing. Particularly, energy has been used successfully for classification application. Thus, the wavelet packet energy representation can provide more robust signal features for classification, while it is difficult to identify these features directly from the expansion coefficients [31,32]. Furthermore, wavelet packet energy can be used to identify the locations and severity of damage. To do that, wavelet packet energy, $E_{n_{j}}$, at $j$ level of node $n$ is defined as follows:

$$
\begin{aligned}
E_{n_{j}} & =\int_{-\infty}^{+\infty} f^{2}(t) d t=\sum_{m=1}^{2^{j}} \sum_{n=1}^{2^{j}} \int_{-\infty}^{+\infty} f_{j}^{m}(t) f_{j}^{n}(t) d t \\
& =\sum_{i=1}^{2^{j}} E_{n_{j}^{i}} .
\end{aligned}
$$

A signal $f(t)$ should be square integrable if $E_{n}$ is finite, that is, $f(t)$ is in Hilbert space $L^{2}(R)$.

Note that energy in this context is not the same as the conventional notion of energy in other areas of science. The signal energy at the $j$ th level and the $i$ th frequency band can be expressed as follows:

$$
E_{j}=\sum_{k=1}^{n}\left|C_{j}(k)\right|^{2}, j=1,2, \ldots \ldots, m .
$$

The total energy of signal is expressed by:

$$
E_{f_{j}}=\sum_{i=1}^{2 j}\left|E_{f_{j}^{i}}\right|,
$$

where wavelet packet component energy, $E_{n_{j}^{i}}$, can be considered to be the energy stored in component signal, $f_{j}^{i}(t)$ :

$$
E_{n_{j}^{i}}=\int_{-\infty}^{+\infty} f_{j}^{i}(t)^{2} d t
$$

Eq. (20) demonstrates that the overall signal energy can be spilt to wavelet packet energy components in various, frequency bands. Finally, the WPERI was developed to identify the location and extent of the damage of the crack [33]:

$$
\Delta\left(E_{f_{j}}\right)=\sum_{i=1}^{2 j} \frac{\left|\left(E_{f_{j}^{i}}\right)_{b}-\left(E_{f_{j}^{i}}\right)_{a}\right|}{\left(E_{f_{j}^{i}}\right)_{a}} .
$$

The term $\left(E_{f_{j}^{i}}\right)_{a}$ is the signal component energy at level $j$ without damage, and $\left(E_{f_{j}^{i}}\right)_{b}$ is the signal component energy at level $j$ with some damage. It 
is assumed that structural damage can influence the wavelet packet component energies and, then, may change this damage indicator. Therefore, it is desired to choose the Wavelet Packet Energy Rate Index (WPERI), because it is sensitive to the alerts in the signal characteristics [34].

\subsubsection{Modified wavelet packet energy}

Structural damage detection utilizing wavelet packet energy is one of the most reliable and efficient structural health monitoring techniques. The energy methods, which are based on strain response data, are rather sensitive compared to those based on acceleration and displacement, because strain energy density involves the second spatial derivatives of the displacement; in addition, it is much more sensitive to small deviation in the structural response than to the displacement itself. Therefore, wavelet packet energy has been modified to include the effect of strain in order to increase the accuracy of damage detection. It is expected that deploying the modified method can obtain more accurate strain energy stored in structural elements; at the end, it provides appropriate, damage detection model and reduces the computation and iteration efforts.

Strain is rather more sensitive to damage than to other raw data, such as deflection, velocity, and acceleration. In certain cases, damage can be directly identified via raw strain data. For structural dynamic responses, the Envelope Area of Strain-time Curvature (EASC) is proposed as a damage index:

$$
S_{n}=\int_{-\infty}^{+\infty} f_{n}(t) d t
$$

To quantify the damage from wavelet packet component energies, the Modified Wavelet Packet Energy Rate (MWPER) is proposed in this paper to detect both the location and severity of the damage. MWPER of node $n$ is defined as follows:

$$
D_{n}=\sum_{i}^{2^{j}}\left[\left[\left(E_{n_{j}^{i}}\right)_{a}-\left(E_{n_{j}^{i}}\right)_{b}\right] \times\left[\left(S_{n}\right)_{a}-\left(S_{n}\right)_{b}\right]\right],
$$

where subscripts $a$ and $b$ stand for damaged and undamaged statuses, respectively.

Wavelet packet energy rate is capable of utilizing information extracted from different frequency-bands, and the envelope area of strain-time curvature extracts the amplitude information from strain time-history data. Thus, MWPER can utilize both the frequency domain information and amplitude information of the raw data.

For a damage identification procedure based on the modified method, it is assumed that the reliable intact and damaged structural models are available, and the structure is excited via the same impulse load and acts at the same location in damage and undamaged structures.

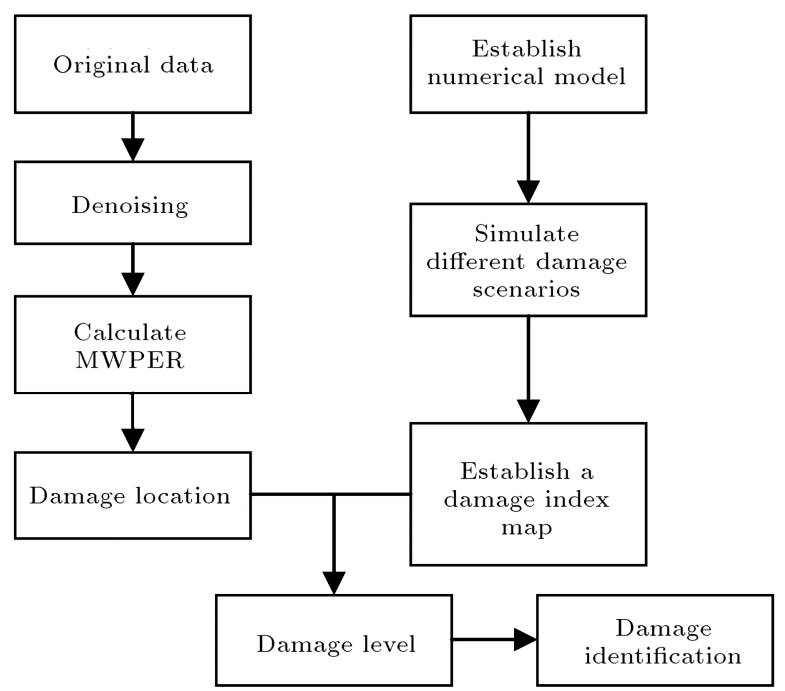

Figure 2. Flow chart of damage identification based on strain-time history data.

\section{Damage identification procedure}

Based on the discussion above, a complete damage identification procedure is established for the second method. Figure 2 shows the complete procedure of damage identification based on WT. The steps for this procedure are described below.

\subsection{Establishment of a numerical model}

Developing a numerical model is the first step to detect the severity of the damage. Thus, a fine mesh numerical model of the structure should be established.

\subsection{Simulation of running car experiments}

At least, four points of damage need to be simulated as described below:

(a) Damage at point DB1 with stiffness loss of SL1;

(b) Damage at point DB1 with stiffness loss of SL2;

(c) Damage at point DB2 with stiffness loss of SL1;

(d) Damage at point DB3 with stiffness loss of SL1.

Based on the MWPER data from simulations (a) and (b) above, the near-linear relationship between the stiffness loss and MWPER can be determined. Based on the MPWER data of (a), (c), and (d), the relationship between the location and MPWER at (a) certain level of damage severity can be determined.

\subsection{Establishment of the damage index map}

Based on the relationships obtained in the previous section, a damage index map is established.

\subsection{Denoising of the input signal}

The original data used in this research represent longgauge strain data obtained via FBG/BOTDR sensors. As discussed before, denoising is commonly the first step for signal processing. Then, all input signals will be denoised by WT. 


\subsection{Calculation of MWPER}

Based on the value of MPWER, the damage location can be found.

\subsection{Combination of the damage location and the value of $M W P E R$}

Since a map is developed where all the relationships have been established, the severity of the damage can be determined by examining the corresponding location and the data from MPWER.

\subsection{Maintenance decision based on the damage location information}

Once the damage location and its severity are verified, it can be determined if the structure needs to be repaired.

\section{Validation by numerical models}

A completed theory for damage identification scheme needs to be validated by experiments. Thus, numerical simulations are essential for the development of the proposed method. The theoretical background has been elaborated in the previous section; hence, numerical simulations are introduced herein to validate this method.

\subsection{Numerical model}

In this section, MWPER is introduced as a damage index, whose calculation is based on WPER and EASC, and the calculation of wavelet packet energy is based on strain time history data of the structure. Since the entire damage identification is based on the strain-time history data, the model used in this section should be able to have enough strain at every part of the structure. Although a simply supported beam will not have any strain at the support points, the supporting condition of fixed ends and simply supported beams can both be considered and compared to see if the proposed method identifies damages in the vicinity of the supports of a simply supported beam. In this work, both beam and single-layer frame models will be used to validate the proposed damage identification.

ANSYS 14.5 is introduced as a numerical simulation platform. For the convenience of modeling, all the material properties and cross-sections are set to be the same among all models. The material properties used herein have been commonly used in numerous research works, including: $E=200 \mathrm{GPa}, \rho=7800 \mathrm{~kg} / \mathrm{m}^{3}$, and $\mu=0.3$, where $E$ stands for Yong's modulus, $\rho$ stands for mass density, and $\mu$ stands for Poisson's ratio.

All three numerical models are described in detail below. For the convenience of comparison and modeling, undamaged and damaged sections of beams and columns in all 3 models are set to be the same. A total of 8 section scenarios are considered in this paper

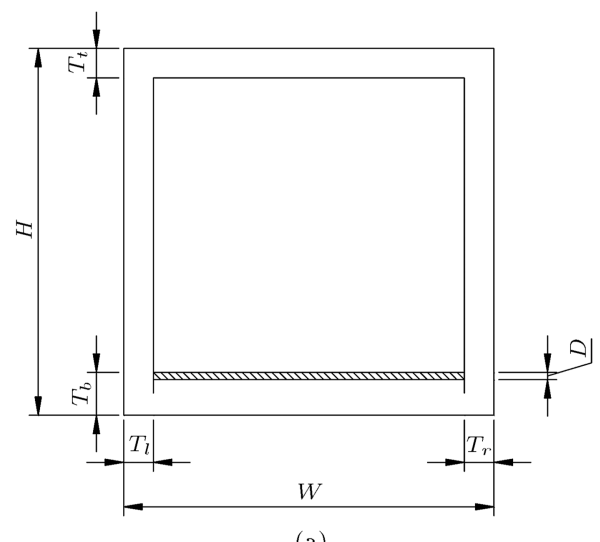

(a)

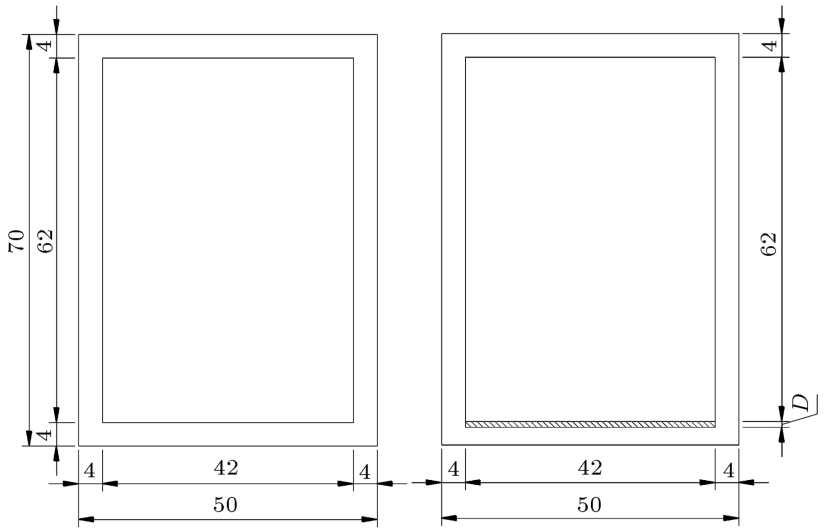

(b)

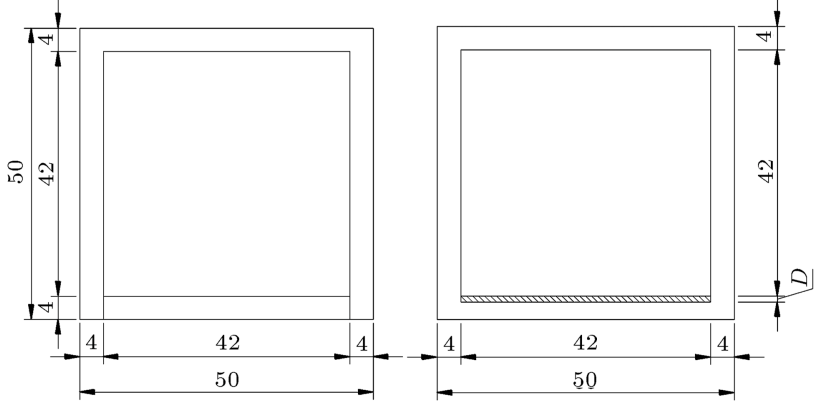

(c)

Figure 3. Sections of undamaged and damaged columns and beams: (a) The section used for the calculation of bending rigidity, (b) sections of undamaged and damaged beam, and (c) sections of undamaged and damaged column.

for the beam model. The beam section is set to be a thin-walled rectangular tube, whose height, width, and thickness are $70 \mathrm{~mm}, 50 \mathrm{~mm}$, and $4 \mathrm{~mm}$, respectively (Figure 3(a)). The column section is set to be a square steel tube, whose height, width, and thickness are $50 \mathrm{~mm}, 50 \mathrm{~mm}$, and $4 \mathrm{~mm}$, respectively (Figure 3(b) and (c)). Corrosion or crack on bridges reduces the working area of the component sections; thus, the damage is simulated by reducing wall thickness. Since hydrops cause corrosion at the bottom wall of a structure, the thickness of bottom wall will be reduced so as to simulate damages. The reduced part of the 
bottom walls is shown in Figure 3(a), (b), and (c) as the shaded parts.

All damages/cracks are simulated by reducing the thickness of the bottom layer. The stiffness loss should be calculated exactly, since the wavelet coefficients might be used as a damage index that are able to reflect the damage severity. For the purpose of simulating the damages with different damage levels, a formula is proposed to calculate the exact thickness loss that is needed for a certain bending rigidity loss. The second moment of area is:

$$
\begin{aligned}
I= & \frac{T_{t}^{3} W}{12}+T_{t} W\left(H_{n}-\frac{T_{t}}{2}\right)^{2}+\frac{\left(T_{b}-d\right)^{3} W}{2} \\
& +W\left(T_{b}-d\right)\left(H-H_{n}-\frac{T_{b}-d}{2}\right)^{2} \\
& +\frac{T_{l}\left(H-T_{t}-T_{b}+d\right)^{3}}{12} \\
& +T_{l}\left(H-T_{t}-T_{b}+d\right) \\
& {\left[\frac{\left(H-T_{t}-T_{b}+d\right)}{2}+T_{t}-H_{n}\right]^{2} } \\
& +\frac{T_{r}\left(H-T_{t}-T_{b}+d\right)^{3}}{12} \\
& +T_{r}\left(H-T_{t}-T_{b}+d\right) \\
& {\left[\frac{\left(H-T_{t}-T_{b}+d\right)}{2}+T_{t}-H_{n}\right]^{2} . }
\end{aligned}
$$

$H_{n}$ and all the parameters, such as $W, H, d, T_{t}, T_{b}, T_{l}$ and $T_{r}$, are shown in Figure $3(\mathrm{~b})$ and (c), where:

$$
\begin{aligned}
H_{n}= & {\left[\frac{W H^{2}}{2}-\frac{(H+d)\left(H+d-T_{t}-T_{b}\right)\left(W-T_{l}-T_{r}\right)}{2}\right] } \\
& /\left[W H-\left(W-T_{l}-T_{r}\right)\left(H+d-T_{t}-T_{b}\right)\right] .
\end{aligned}
$$

With this formula, a damage ranging from zero to $30 \%$ bending rigidity loss is proposed for the following sections. Table 1 shows the exact thickness loss of the bottom layer.
Table 1 shows all beam section information listed in column sections; only one damage severity level is used, and the stiffness loss of that damage is $3.81 \%$.

$D$ represents the section plots (Figure $3(\mathrm{~b})$ and (c)) which stand for damage depths; for the convenience of description, zero damage depth means that there is no damage at the corresponding section (Section ID 1 as described in Table 1).

The damage location information for the adopted numerical models introduces the following detail:

- Model 1: Simply supported beam. The simply supported beam's length is set to be $1000 \mathrm{~mm}$, with a damage introduced at distances of $D B$ from the left support as shown in Figure 4(a).

- Model 2: Fixed ends beam. The fixed ends beam's length is set to be $1000 \mathrm{~mm}$, which is the same as the simply supported beam's. The damage location parameter, $D B$, is defined as the distance

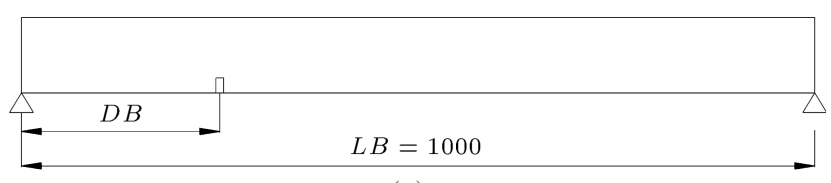

(a)

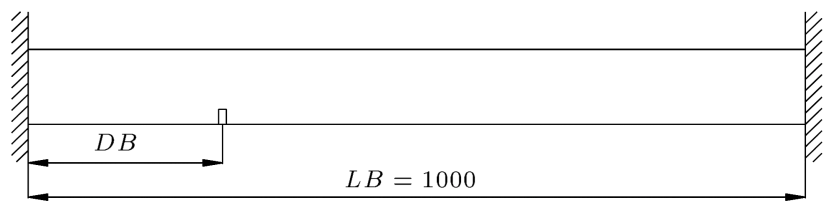

(b)

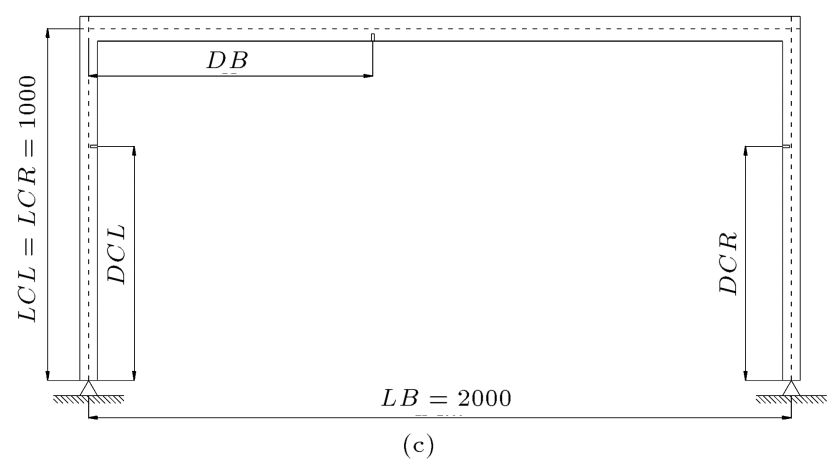

Figure 4. Detail of The numerical models: (a) Simply supported beam model, (b) fixed ends beam model, and (c) single layer frame model.

Table 1. Section information of all the sections used in this work.

\begin{tabular}{ccccccccc}
\hline Section type & \multicolumn{7}{c}{ Beam section } \\
\hline Section ID & 1 & 2 & 3 & 4 & 5 & 6 & 7 & 8 \\
Area $\left(\mathrm{mm}^{2}\right)$ & 896 & 875 & 867 & 840 & 816 & 794 & 774 & 755 \\
Damage depth $(\mathrm{mm})$ & 0 & 0.5 & 0.6972 & 1.3252 & 1.8964 & 2.4202 & 2.9037 & 3.3525 \\
IY (10E6 mm $\left.{ }^{4}\right)$ & 5.95 & 5.74 & 5.65 & 5.36 & 5.06 & 4.76 & 4.46 & 4.17 \\
Stiffness loss (\%) & 0 & 3.52 & 5 & 10 & 15 & 20 & 25 & 30 \\
\hline
\end{tabular}


between the damage location and the left support, as shown in Figure 4(b).

- Model 3: Single layer frame. The single layer frame shown in Figure $4(\mathrm{c})$ is used as the 3rd numerical model. Damages are marked by rectangular symbols in Figure $4(\mathrm{c})$, and $D B$ is the distance of the damage location from the centroidal axis of the left column. $L B$ is the total length of the beam. $D C L$ is the distance from the centroidal axis of the left column, while $L C L$ is the total length of the column. The unit used in Figure 4(c) is $\mathrm{mm}$.

\section{Damage scenarios}

To establish the second method, several different damage scenarios need to be taken into consideration. There are three cases for the establishment and validation of that method.

Firstly, two different kinds of excitations are compared, simulated via contact elements in ANSYS. So far, most dynamic experiments are done by hammer excitation. For bridges, cars run on the top of the beam. Both running car excitations and hammer excitations are usually used for dynamic experiments on bridges; thus, these two types of excitations are adopted in this work. The hammer is simulated by $8 \mathrm{~kg}$ mass, and it hits the structure at a speed of $4 \mathrm{~m} / \mathrm{s}$. The running car is simulated by $8 \mathrm{~kg}$ mass and the car runs from the left side to the right side at a speed of $2 \mathrm{~m} / \mathrm{s}$.

Secondly, the situation in which damages occur at points where the strain is relatively small must be taken into consideration to validate if the proposed method can be used independently to detect without additional detection method. As mentioned in the previous sections, the proposed method only works in the context in which strain exists in every part of the structure. For steel bridges, although stain exists everywhere on the structure, there are always some points where the strain is relatively small, making it essential to validate the proposed method for smallstrain situation cases.

Thirdly, a multiple-damage scenario must be taken into consideration, since the damages/cracks on steel bridges may occur simultaneously. Although the theory behind the second damage identification method indicates that the spatial resolution of the identification is limited by the density of sensors and the sensing range of each sensor, the multiple-damage scenario must be validated to ensure the viability and compatibility of the method with steel bridges.

Finally, the single layer frame with damages to columns must be tested, since the excitation range is limited within the main beam. While columns are not directly excited by excitations, this damage scenario must be considered and validated.
Based on the discussion above, four damage scenarios are considered and used to establish and validate the second method. For the convenience of analysis, the sensor interval is set to be the same as element size, and the default element size is set to be $12.5 \mathrm{~mm}$. To identify the effect of sensor interval, there are three element sizes in Scenario 2: $12.5 \mathrm{~mm}, 50 \mathrm{~mm}$, and $200 \mathrm{~mm}$, meaning that there are 80,20 , and 5 sensors on the beam of Scenario 1, respectively.

1. Hammer excitation on a fixed end beam with one damage at position $D B=0.25 \mathrm{~m}$;

2. Running car excitation on a fixed end beam with one damage at position $D B=0.25 \mathrm{~m}$ and the car running from the left support to the right support at a speed of $2 \mathrm{~m} / \mathrm{s}$;

3. Running car excitation on a simply supported beam with one damage at positions $D B=0.025 \mathrm{~m}$ and $D B=0 \mathrm{~m}$. The car runs from the left support to the right support at a speed of $2 \mathrm{~m} / \mathrm{s}$. Since numerical models consist of many elements, the so called $D B=0 \mathrm{~m}$ is simulated by the section change of the element on the left side of the beam;

4. Running car excitation with 2 damages to the main beam of a single-layer frame model and one damage to the left front column. The damages occur at $D B 1=0.5 \mathrm{~m}, D B 2=0.1 \mathrm{~m}$, and $D C L=0.5 \mathrm{~m}$. The speed of the running car is $4 \mathrm{~m} / \mathrm{s}$, and the car runs from the left end of the main beam to the right end of the main beam.

For all scenarios, the strain data of different sensors are recorded simultaneously. The record for Scenario 1 begins at 0.5 before the excitation and lasts for 5 seconds. The record time for Scenarios 2 and 3 is 4.5 seconds, and the record starts when the car starts to move from the left support. For Scenario 4, the record starts when the car starts to move from the left support and, also, lasts for 4.5 second.

\subsection{The establishment of the proposed method} Based on the damage identification theory proposed previously, several issues must be taken into consideration and modified to establish a reliable damage identification scheme. Based on this idea, the following parts are analyzed to refine the damage identification theory into a complete damage identification scheme.

In this section, Damage Scenario 1 is used to present the effectiveness of MWPER by comparing Modified Wavelet Packet Energy Rate (MWPER), Wavelet Packet Energy Rate (WPER), and Envelope Area of Strain-time Curvature (EASC).

\subsubsection{Comparison of the proposed damage index and conventional indices}

A single damage with $3.52 \%$ stiffness loss is considered, and the damage is assumed to be at the 20th and 21th 


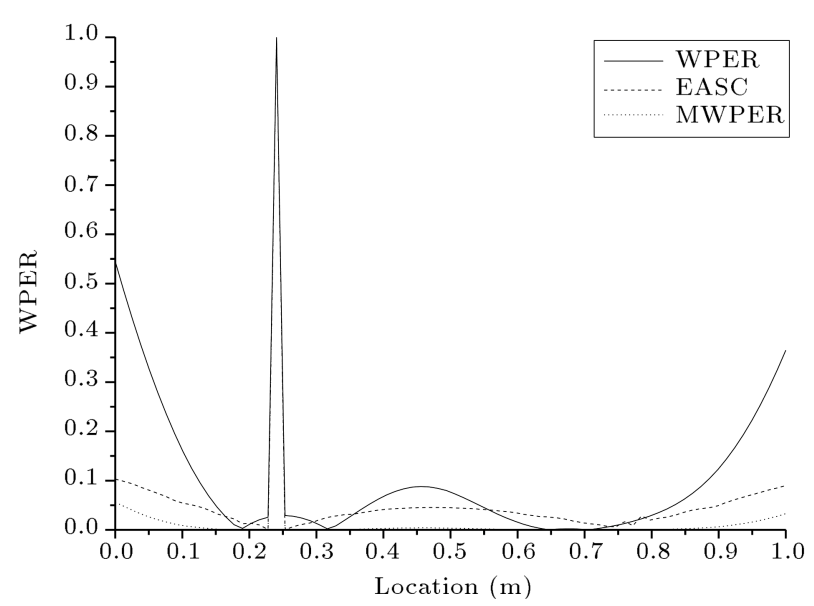

Figure 5. Comparison of WPER, EASC, and MWPER (normalized).

elements, which are located $0.25 \mathrm{~m}$ away from the left support. All 3 damage indices are normalized to 1 for the purpose of making the peak values the same (Figure 5).

All 3 damage indices are capable of indicating the existence of damage in Scenario 1. Values of WPER and EASC are much larger than that of MWPERR on elements which are intact, especially on elements near the supports, indicating that WPER and EASC are not so stable when applied on small-damage cases and may lead to a false indication of damage in some cases where damage severity is low. Compared with WPER and EASC, MWPER is stable with disturbance less than 0.05 on intact elements.

\subsubsection{Comparison between hammer excitation and running car excitation}

In this section, Scenarios 1 and 2 are used to choose the best excitation mode for the proposed damage identification. All damages are set to be $D B=0.25$, and the stiffness loss is set to be $3.52 \%$. For hammer excitation, the hammer hits the beam at a speed of $4 \mathrm{~m} / \mathrm{s}$ at time $0.575 \mathrm{~s}$, and the recording lasts for 4.5 seconds. Figure 6 shows the strain-time history data of the midpoint. Figure 7 shows the instantaneous strain data of the entire beam at $0.01 \mathrm{~s}$ after the excitation by the hammer.

For running car excitation, the recording starts when the car starts to move from the left support and ends at 3.925 seconds after the car leaves the right support. The recording lasts for 4.5 seconds, the same as the hammer excitation. Figure 8 shows the straintime history of the midpoint of the beam. Figure 9 shows the instantaneous strain of the fixed end beam at $0.01 \mathrm{~s}$ after the car runs off the main beam.

Figure 10(a) shows the comparison between hammer excitation and running car excitation. Results show that the running car result is much better than the hammer excitation, especially at the supports.

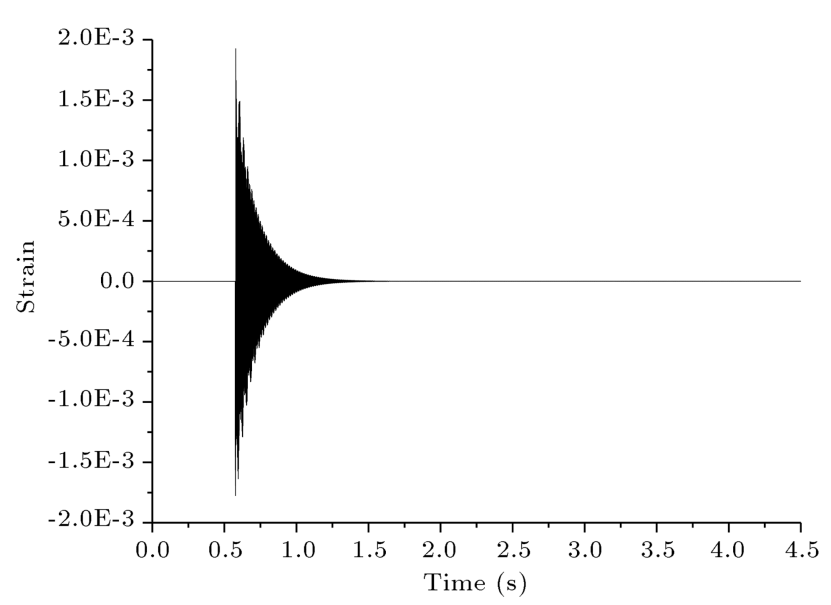

Figure 6. Strain-time history data of hammer excitation.

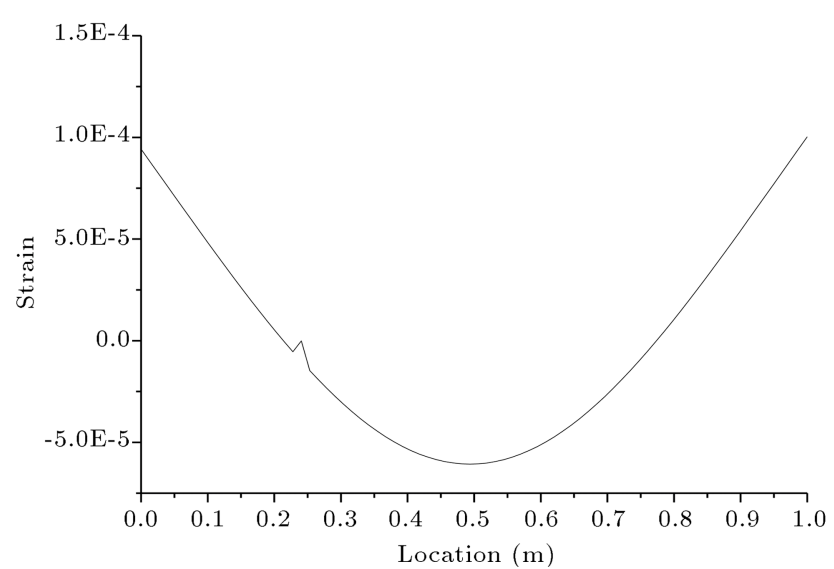

Figure 7. Instantaneous strain at time $0.585 \mathrm{~s}$ of hammer excitation.

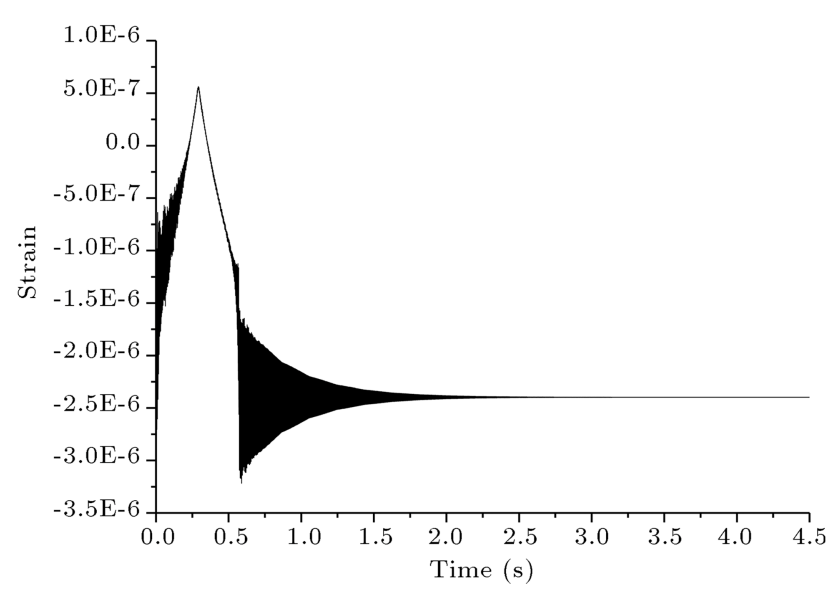

Figure 8. Time history data of running car excitation.

While the damage index of the hammer excitation reaches 0.05 at the supports, that of the running car excitation is almost zero, which means that the MWPER of a running car excitation is more stable than that of a hammer excitation.

The above-mentioned phenomenon is caused by the number of excitation points. The proposed method 


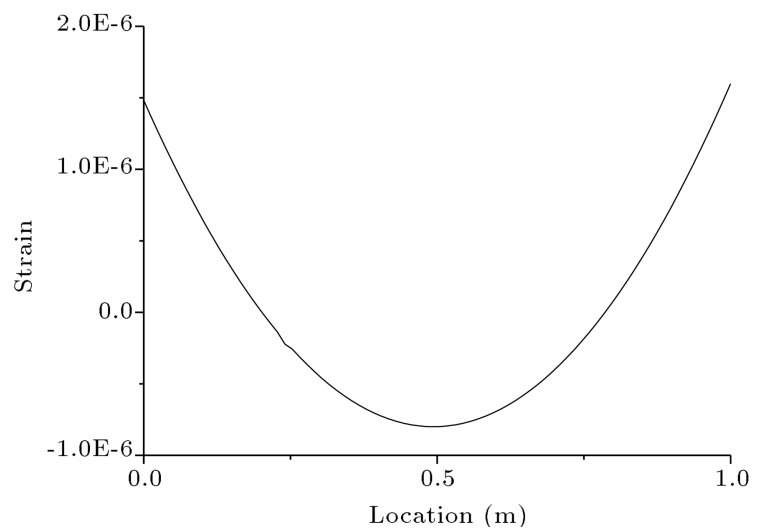

Figure 9. Instantaneous strain at time $0.585 \mathrm{~s}$ of running car excitation.

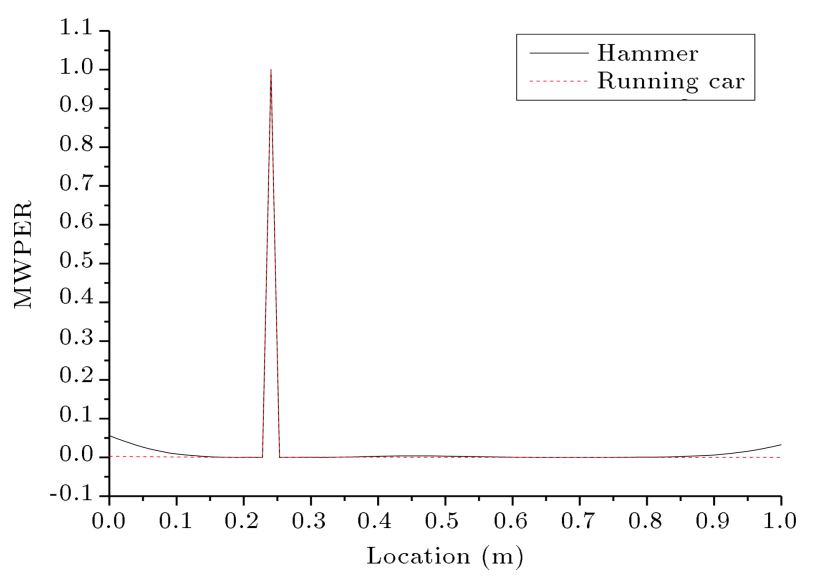

(a)

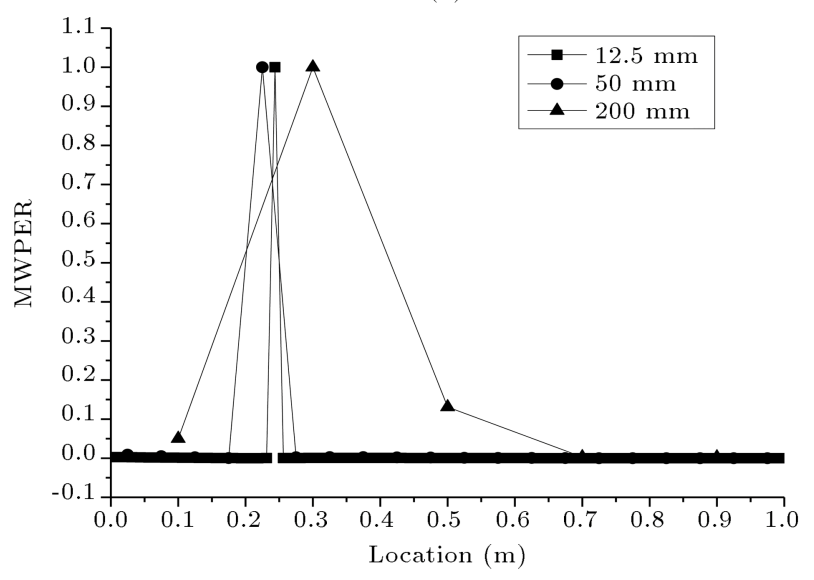

(b)

Figure 10. Comparison between hammer excitation and running car excitation.

is capable of combining all the strain-time history data into one index for damage identification, meaning that the more points with larger strain exist, the more stable result we can obtain. With a running car excitation, every part could be excited, resulting in a large number of points with large strain, meaning better damage identification. Thus, for damage identifications in a steel bridge, it is best that cars run from the left end to the right end, i.e., the entire span of the beam, in order to collect enough response data.

\subsubsection{Effects of different sensor intervals}

In this section, Scenario 2 is used to examine the effect of sensor interval on the proposed damage identification. All damages are set to be $D B=0.25$, and the stiffness loss is set to be $3.52 \%$. There are three sensor intervals, i.e., $12.5 \mathrm{~mm}, 50 \mathrm{~mm}$, and $200 \mathrm{~mm}$, meaning that each sensor covers $12.5 \mathrm{~mm}, 50 \mathrm{~mm}$, and $200 \mathrm{~mm}$ lengths of the beam, respectively.

As Figure 10(b) shows, the proposed damage index is capable of identifying damage with five sensors on the beam, where the sensor interval is $200 \mathrm{~mm}$. The identified damage locations are different. With $200 \mathrm{~mm}$ sensor intervals, the identified damage location is about $300 \mathrm{~mm}$ from the left support; with 50 $\mathrm{mm}$ sensor intervals, the identified damage location is about $225 \mathrm{~mm}$ from the left support; with 12.5 $\mathrm{mm}$ sensor intervals, the identified damage location is about $250 \mathrm{~mm}$ from the left support. While the actual damage location is $D B=250$, the damage location identification errors can be explained and are acceptable. This phenomenon is caused by the sensors' mechanism. For long-gage fiber optical sensors, the strain obtained is the average strain of the whole measure length, which means that as long as the damage location covered by sensors is applied, the strain change can be captured by the corresponding sensor. Since the $x$ coordinate for each data point in Figure 10(b) is set to be the mid-point of corresponding sensor coverage length, the peak in Figure 10(b) means that there is a damage or damages in the corresponding sensor interval. Under the context that only 5 sensors or 20 sensors set up on the beam, Figure 10(b) shows that the proposed method is capable of identifying damages with only a few sensors.

\subsubsection{Detectability of damages nearby the supports of the simply supported beam}

In this section, Damage Scenario 3 is used to test if the second damage identification method is able to detect damages near the supports of a simply supported beam. The second damage identification method is based on strain signals. Since the strain values at the points near the supports of a simply supported beam are much lower than those at the points in the middle of the span, it becomes the main concern if damages that occur at points where the strain signals are weak can be identified.

Stiffness loss of $3.52 \%$, the same as those in the previous sections, is introduced on the simply supported beam. The car is run from the left side to the right side at a speed of $2 \mathrm{~m} / \mathrm{s}$. One damage is set to the left end of the simply supported beam. Another damage is set at a distance of $0.025 \mathrm{~m}$ from 


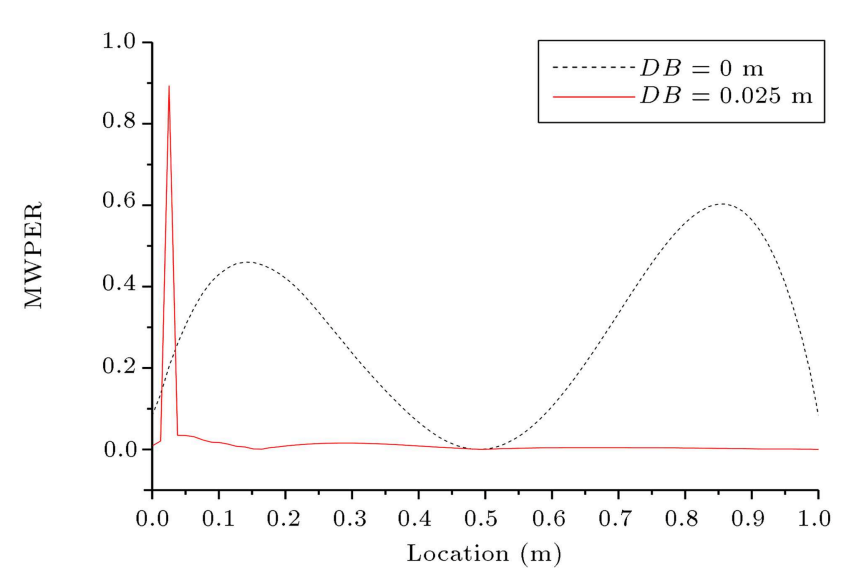

(a)

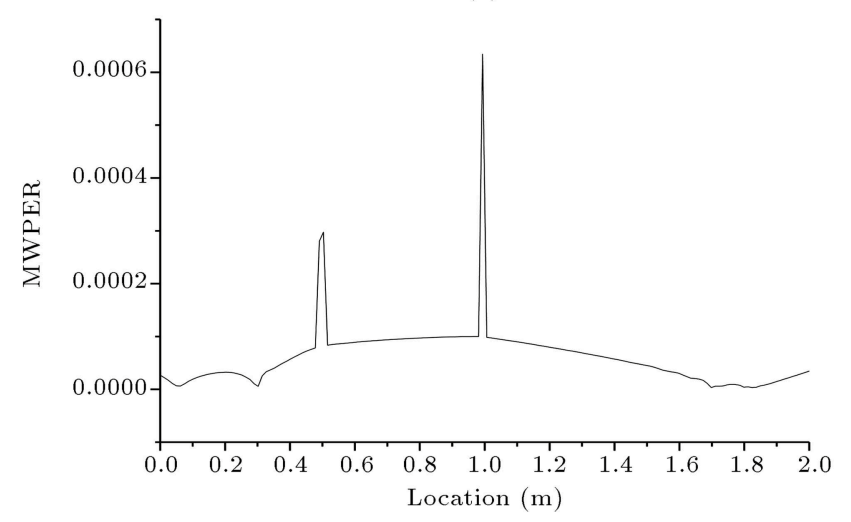

(b)

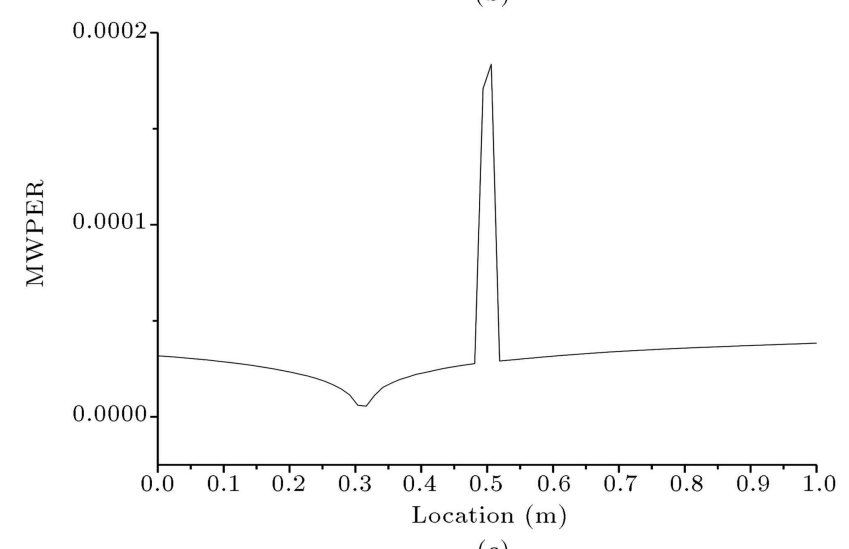

(c)

Figure 11. The normalized MWPER of beams: (a) Damage at $D B=0 \mathrm{~m}$ and $D B=0.025 \mathrm{~m}$, (b) the main beam with two damages, and (c) left front column.

the left support. Figure 11(a) shows that the damage at $D B=0.025 \mathrm{~m}$ can be identified, while the damage at $D B=0 \mathrm{~m}$ cannot be identified.

All the MWPERs are normalized in order to best estimate the damage location. Thus, the absolute values shown in Figure 11(a) are not of concern to us; only the relative values need to be of concern. The reason behind the fact that the strain in the elements near the supports of a simply supported beam is not totally zero, while strain of the elements at the support is almost zero, implies that all data in Figure 11(a) are normalized. With FBG/BOTDR strain sensors, the results that are obtained represent the information gathered from the entire area covered by the sensors. Therefore, the strain change, caused by the damage, at $D B=0.025 \mathrm{~m}$ is detectible, while that of the damage at $D B=0 \mathrm{~m}$ is covered by other parts, whose strain value is much larger.

Since damages close to the hinge support can be identified, it can be concluded that as long as the damaged area is covered by a long-gauge strain sensor, and the strains on that area are not all zero, damages located at small strain areas can be identified. However, the damage will not be detected if the strain is too small, such as the strain on the elements located at the supports of a simply supported beam.

\subsubsection{Detectability of multiple damages to the main beam and the damage to the column}

In the next step, we explore if the concept behind the second method can be validated by applying this approach to multiple damage situations. In this section, this proposed concept is validated by the single layer frame model with two damages to the main beam.

Since the running car excitation can only be applied to the main beam of the single layer frame, meaning that the columns cannot be excited directly, we will explore how damages to the columns can be taken into account and detected. Damage Scenario 4 is used to test if the proposed method can be used for this case or not.

Figure 11(b) and (c) show that all three damages are identified. Figure 11(b) indicates that multiple conditions are applicable for MWPER, which is predictable since the strain is sensitive to local damage. Figure 11(c) shows that damages to a component that is not directly excited, i.e., the left column, are also detectable for MWPER. Thus, MWPER is an effective detection tool for application in steel bridges since all damages to all components can be identified.

\subsubsection{Verification of the noise effect on the proposed method}

All the results presented in this section are based on strain-time history data obtained from the finite element analysis of the response; hence, they contain no experimental noise. For real cases, experimental noise is inevitable. To evaluate the robustness of MWPER under measurement noise, the simulated data of Scenario 4 are contaminated with a certain level of artificial random noise to generate 'measured' data. Normally, distributed random noises, whose amplitudes are $5 \%, 10 \%, 30 \%$, and $50 \%$ of the RootMean-Square (RMS) value of strain data, respectively, are added to the strain-time history data.

MWPERs in Figure 12(a) to (d) are normalized to make the comparison clear. With the increase of the 


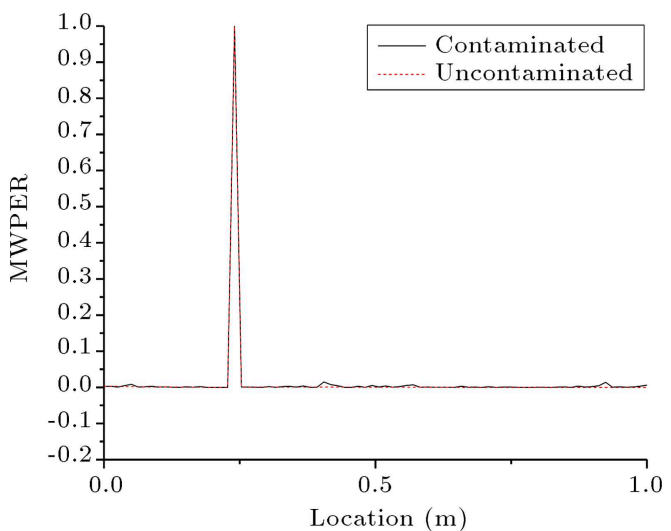

(a)

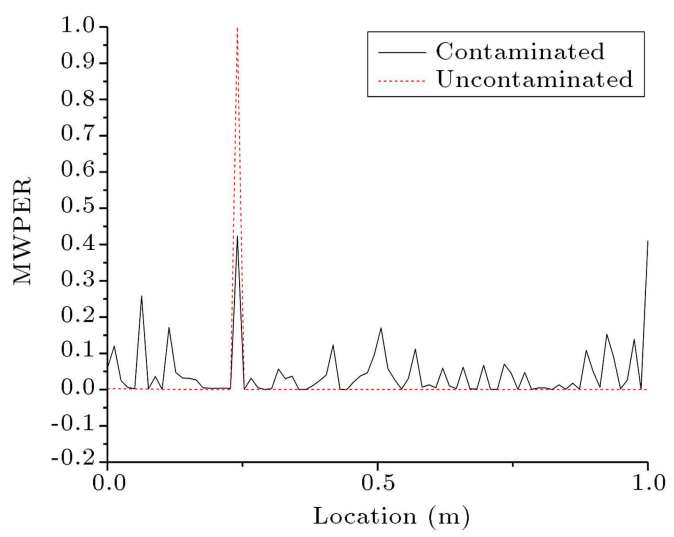

(c)

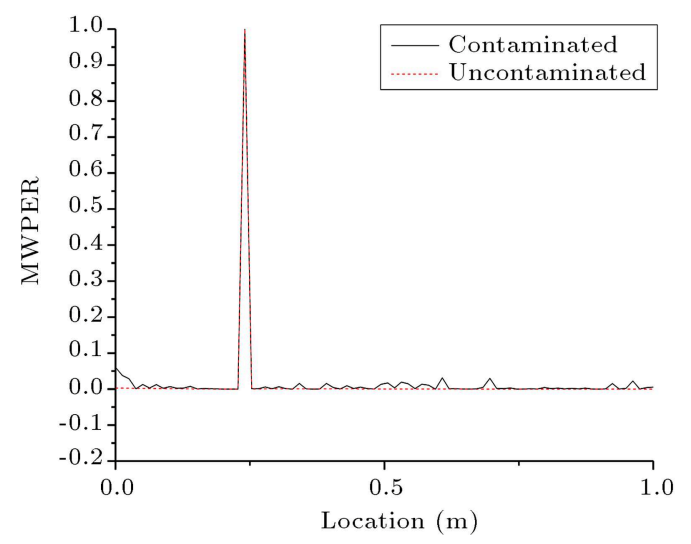

(b)

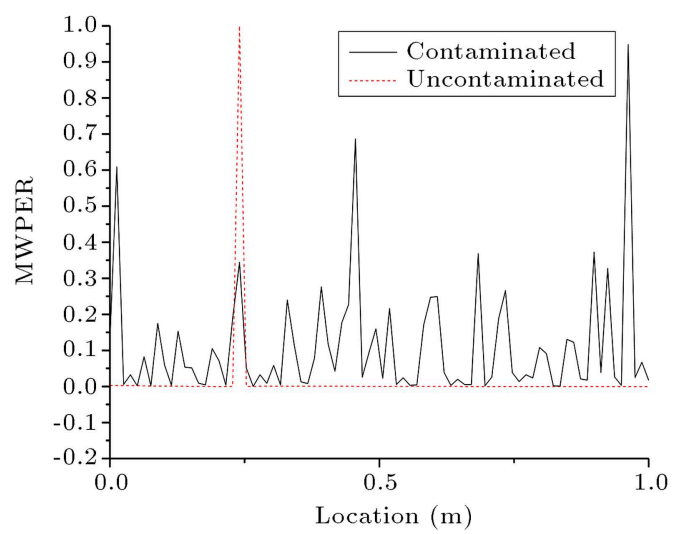

(d)

Figure 12. Normalized MWPER with different contaminated data: (a) Contaminated data (5\%), (b) contaminated data $(10 \%),(c)$ contaminated data $(30 \%)$, and (d) contaminated data $(50 \%)$.

noise level, disturbance on intact elements increases, especially on elements near the end points of the beam. It is important to note that even under a noise level of $30 \%$, the damage can still be identified. This demonstrates that the second damage identification method is robust enough to take into account the measurement noise.

\subsubsection{Effects of stiffness loss level}

As stated in the previous sections, MWPER is capable of identifying the location of a damage under a certain noise level, which means the 2nd level of damage identification. In order to accomplish the 3rd level of damage identification and to show the ability of MWPER to quantify the damage, in this section, MWPERs of different stiffness loss levels $(3.52 \%, 5 \%$, $10 \%, 15 \%, 20 \%, 25 \%$, and $30 \%$ ) are discussed.

Figure 13 shows the MWPERs on the 20th element changes with the change of stiffness loss. As observed, the MWPERs also show a near-linear relationship with the stiffness loss. Thus, with more stiffness loss level of finite element data, MWPER is capable of identifying stiffness loss level of a specific position. For a damage identification method to detect both the location and severity of a damage,

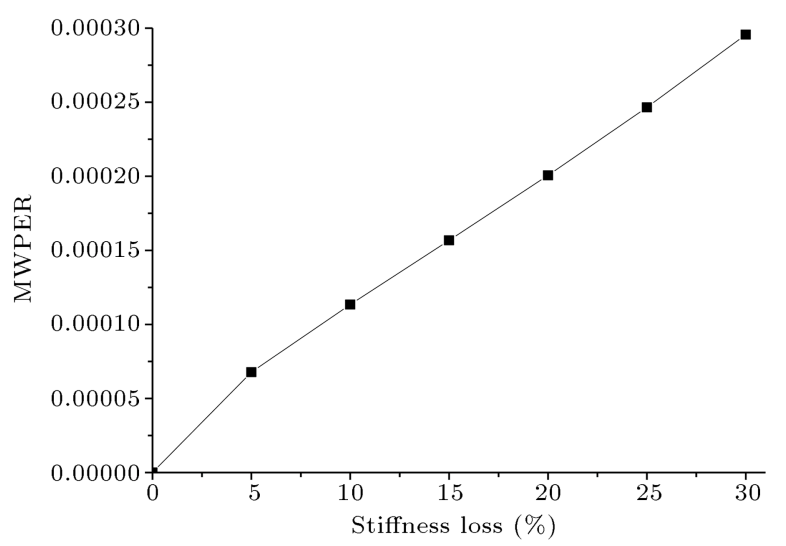

Figure 13. MWPERs of different stiffness loss levels.

the relationship between the damage index and the location needs to be clarified. As demonstrated in this case, the relationship between the location and the value of MWPER is estimated. Therefore, this approach is capable of detecting the severity of the damage, too.

\subsubsection{Effects of damage location}

Quantification of the damage needs a precise numerical relationship between the damage severity and the dam- 
age index. In the previous section, it was verified that MWPER had a near-linear relationship with stiffness loss. Therefore, the effects of the damage location need to be taken into consideration. In this section, Damage Scenario 2 is considered in order to explore and discuss the effects of damage location with the damage simulated by a stiffness loss of $3.52 \%$.

Since both the damage location and the damage level affect MWPER, the effects of damage location must be determined in order to make this damage identification method capable of determining damage level. Subsequently, the damage spanned across locations $D B=0$ to $D B=1$ is simulated to determine the exact effects of the damage location on MWPER. Eighty damage locations were simulated to obtain the acquired results. Figure 14(a) shows the MWPER of damaged beams with damage locations spanning across $D B=0$ to $D B=1$.

\subsubsection{Consideration of both damage location and damage level}

In this section, both a simply supported beam and a fixed end beam are used to calculate the relationship map among the location, damage severity, and location. Eighty damage location situations and 6 levels of stiffness loss are considered, meaning that each beam's numerical model needs to be simulated for $80 * 6=480$ times. In addition, there are two support conditions leading to $240 * 2=480$ times of simulation. This requires an exhaustive computational time. Thus, to utilize ANSYS for this computation, a computation platform is set up, which consists of an Intel 4790K, 16GB DDR3 $2200 \mathrm{MHz}$, and a RAID0 disk array, in order to carry out a fast computation. Finally, these simulations form two damage index maps that can be used to identify damage severity.

As can be seen in Figure 14(b) and (c), the support conditions affect the distribution of MWPER. Thus, for different structures, different MWPER maps need to be established for the purpose of damage severity identification.

\subsubsection{Consideration of modeling error}

As discussed in the previous section, the identification of damage level needs a MPWER map generated by FEM simulation. The modeling-error must be taken into consideration, especially for its effect on the accuracy of damage severity identification. Based on Scenario 2, 6 modeling errors are tested. The stiffness of the simulated beam changes to $80 \%, 90 \%$, $110 \%, 120 \%, 130 \%$, and $150 \%$ of the original stiffness and, then, is tested. The result MWPERs are shown in Figure 15. The MWPER has a nearly linear relationship with the stiffness change, almost the same as the index-damage relationship. The similar nearly linear relationships can be explained by the mechanism

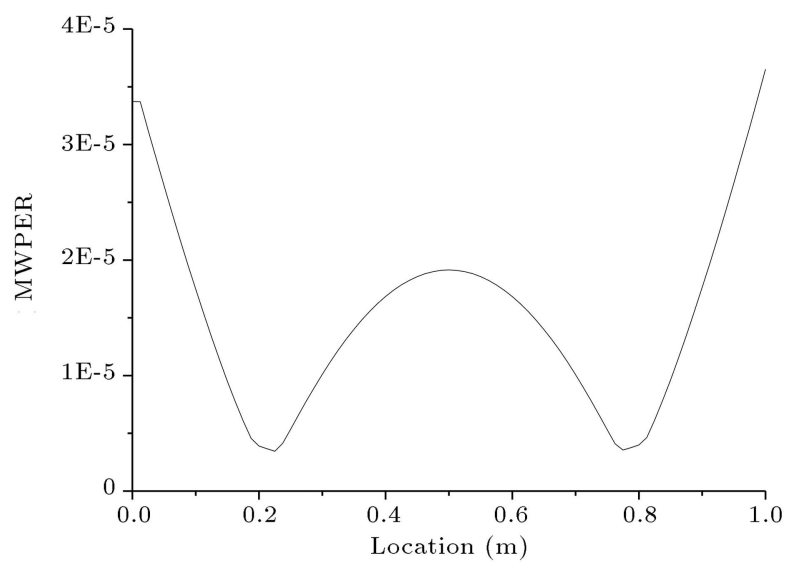

(a)

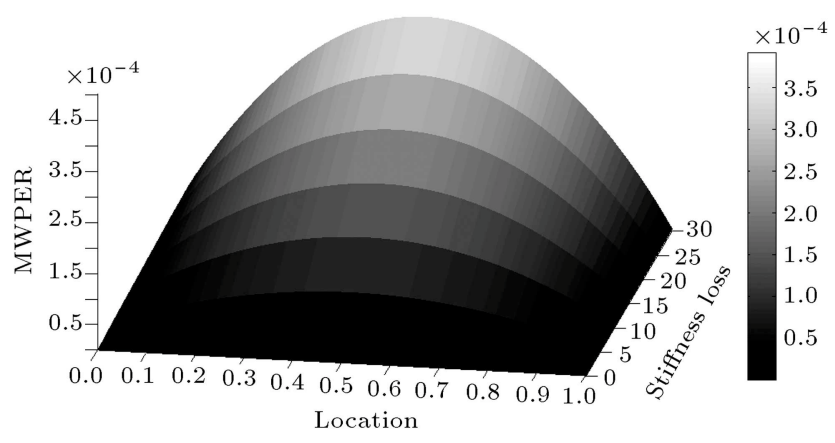

(b)

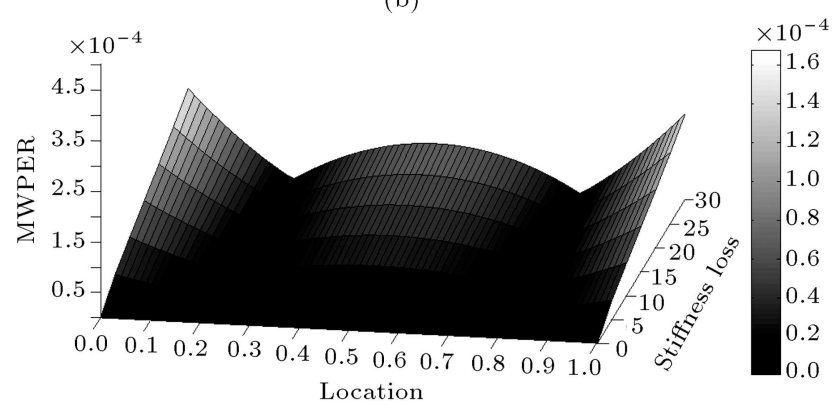

(c)

Figure 14. MPWER of different damage scenarios: (a) Damage locations spanning across $D B=0$ to $D B=1$, (b) the simply supported beam, and (c) the fixed ends beam.

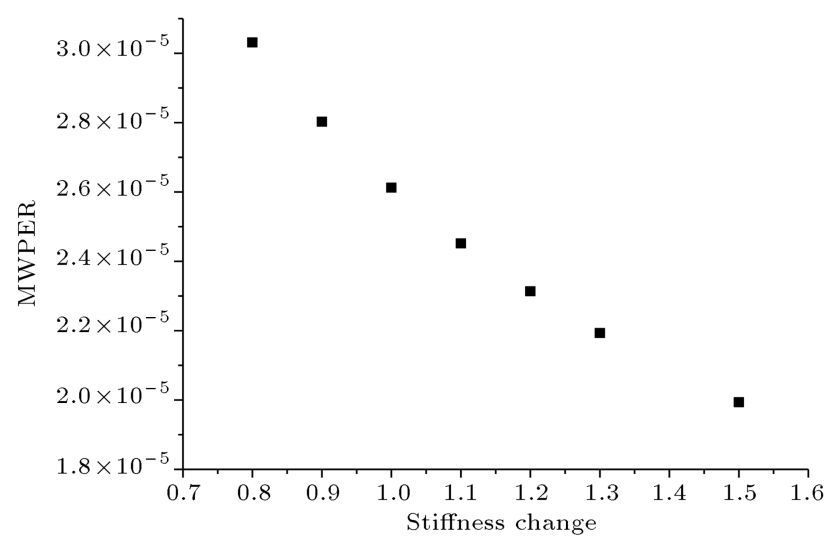

Figure 15. MPWER of different damage scenarios on the fixed ends beam. 
of the proposed method. Both damage or modeling error will lead to the change of strain signal. In addition, their effects on strain amplitude are almost of the same degree, meaning that both the strain change caused by damage and that caused by modeling error will lead to the increase of MWPER.

Figure 15 and the discussion above show that modeling error has large impact on the accuracy of the proposed method. The modeling error must be limited with a small interval to ensure that the damage severity identification result is accurate.

\subsubsection{Validation by a scaled single layer frame}

In this section, Scenario 4 is used to validate the proposed damage identification scheme. As stated in previous sections, by utilizing MWPER, location of all damages can be identified. Thus, the only information that needs to be obtained and validated is the capability of this approach in identifying the damage severity.

MWPER values of two damages to the main beam will be used as the reference to establish a damage index map. Subsequently, the index map will be utilized to identify the severity of the damage to the left front column.

Figure 16 shows the damage index map established for the left front column. As can be seen, its shape is between a simply supported beam and a fixed ends beam. Based on the damage index map, the severity of the damage to the column can be identified. As noted earlier, the MPWER of the damage point on the column is $1.836 \mathrm{E}-4$ and the damage location is at $D L C=0.5 \mathrm{~m}$ (Figure 15). Based on these two coordinates, the third coordinate in Figure 16 can be found, which is $22.36 \%$. While the stiffness loss in simulation is set to be $21.35 \%$, the stiffness loss value obtained via the MWPER map is $22.36 \%$. Thus, the damage severity can be identified by MPWER.

\section{Validation by experiment}

The best way to determine if a damage identification method is good is always by applying it to a real struc-

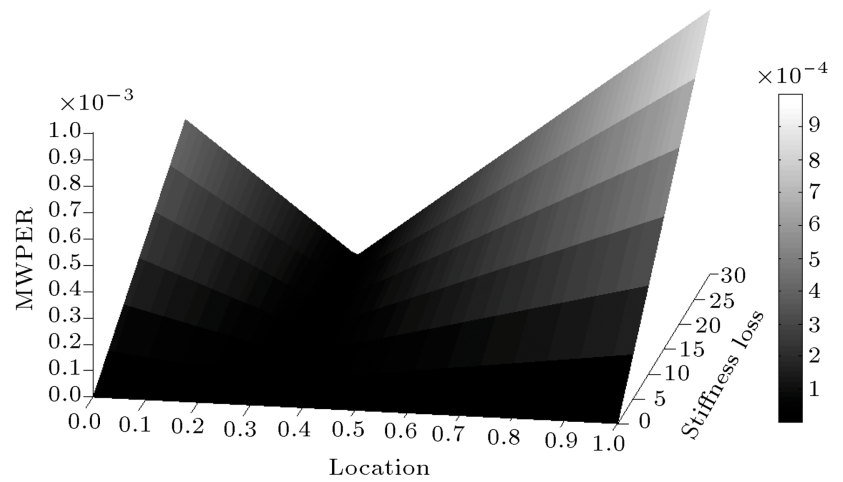

Figure 16. Damage index map for left front column. ture. Thus, in this work, an experiment is designed and run to determine whether the damage identification method works well or not. The experimental result showed that the proposed method was capable of identifying the damage in a real structure.

\subsection{Design of the experiment}

In order to validate the effectiveness of the proposed damage identification method, a simply supported steel beam is set up. The steel beam is an H-shape steel beam. The section of the bean is shown in Figure 17, where the length unit is chosen as $\mathrm{mm}$. The length between two supports is $6000 \mathrm{~mm}$ (Figure 18(a)).

Usually, the fixed ends supporting condition is difficult to reach, limited by the fixation method. Besides, a simply supported support condition will lead to lower strain values nearby the supports, which is helpful for testing the efficiency of the proposed method. Hence, a simply supported beam is set up as the main structure of this experiment.

As discussed earlier, commonly used longgage strain sensors include FBG sensors and BOTDER/BOTDA sensors. Both of them are capable of acquiring long-gage strain information from the structure. FBG sensors enjoy better accuracy in both dynamic and static sensing, while the sensing range limits FBG sensors. BOTDR/BOTDA sensors are suitable for long range sensing, such as a range of over several kilometers. However, the accuracy of BOTDR/BOTDA sensors is lower than FBG sensors. Considering that the steel beam is only 6 meters long, FBG sensors are used in this experiment.

The sampling rate is usually determined by the natural frequencies of the tested structure and sensing system properties. FBG sensors' sampling range can reach an extremely high value; thus, no limitation is placed on the decision of sampling rate. Then, the natural frequencies of the tested structure should

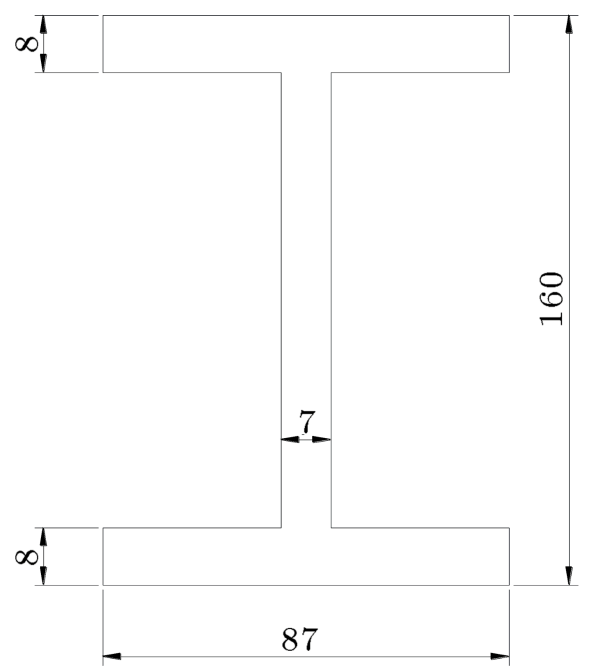

Figure 17. Section information of the tested beam. 


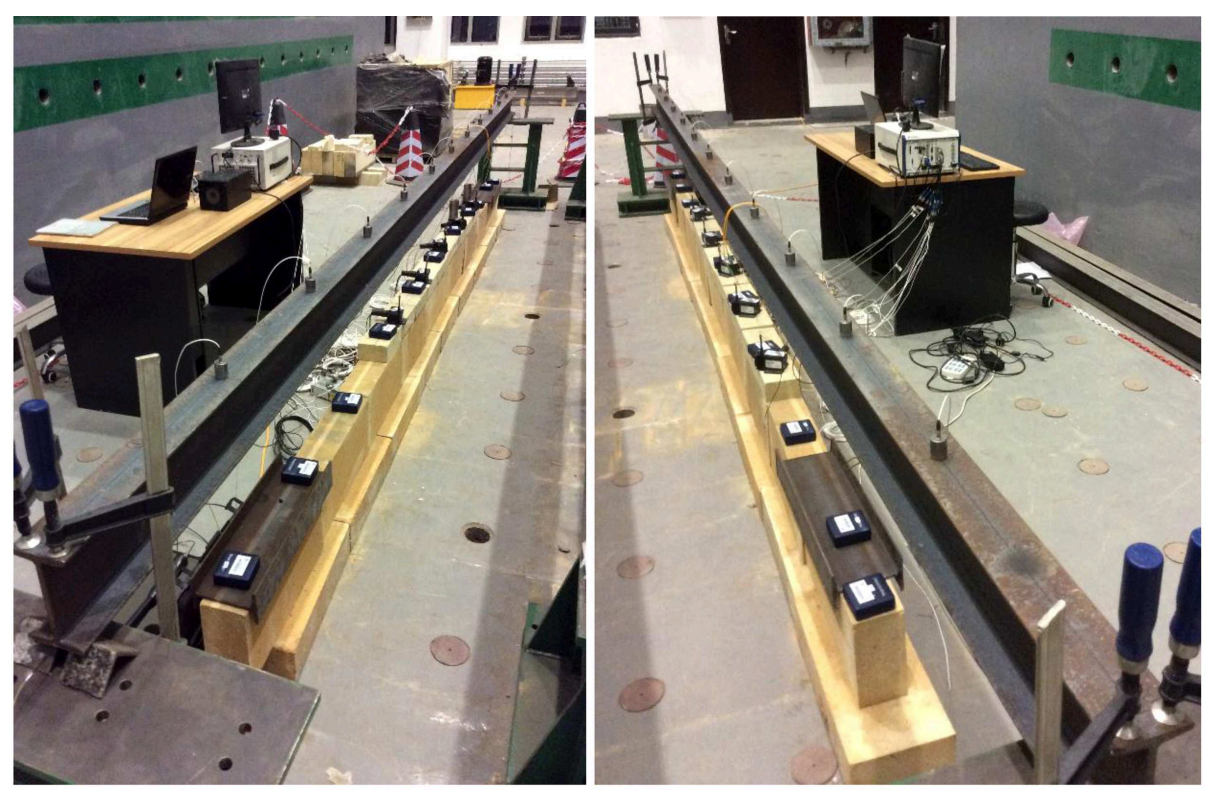

(a)
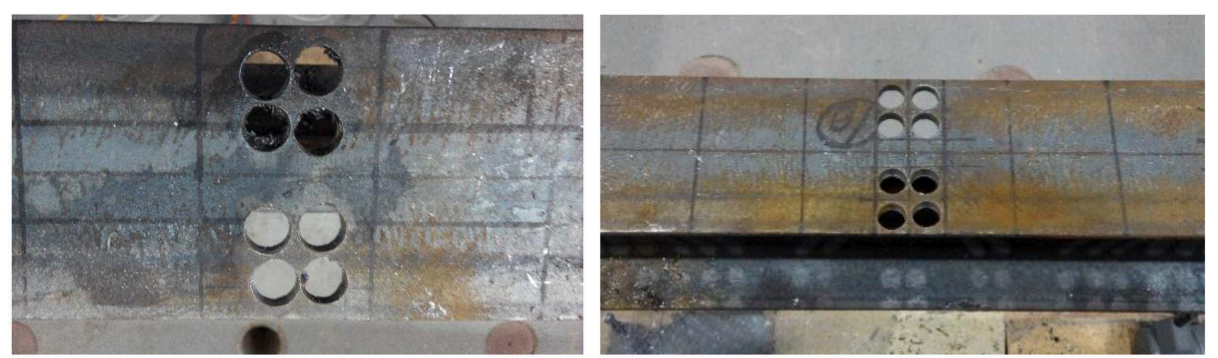

(b)

Figure 18. Design of the experiment: (a) The tested beam with sensors on both the top surface and bottom surface, and (b) damage introduced on top surface.

be considered as the only aspect that determines the sampling rate. According to numerical experiments, the first three natural frequencies include $6.51 \mathrm{~Hz}$, $10.65 \mathrm{~Hz}$, and $31.94 \mathrm{~Hz}$. Since the first three natural frequencies are the most important for analysis, the sampling rate is set to be $1000 \mathrm{~Hz}$, which is high enough to capture all vibration features of the beam.

This experiment aims to obtain sufficient data. Hence, sensors, such as accelerometers, displacement meters, and FBG strain sensors, are installed on the tested beam. For the convenience of installation, FBG sensors are pasted on the bottom surface with displacement meters, while accelerometers are installed on the top surface of the tested beam. As can be seen in Figure 18(a), many sensors are distributed on the top and bottom surfaces of the tested beam. Thus, running car excitation is impossible for this tested beam. As a result, point excitation is adopted in this experiment. The excitation is introduced by a force hammer, which helps record the hammer force for the experiment.

In this experiment, the damage to the beam is introduced by cutting holes at top flange (Figure 18(b)). Since these holes cannot be cut accurately, the stiffness loss of the damage section is calculated after the cutting procedure. By accurate measurement and calculation, the stiffness loss of the damaged section is determined to be $6.6 \%$.

\subsection{Experiment process}

The FBG sensors used in this experiment have a sensing range of $1.5 \mathrm{~m}$. Based on sensor parameters, number of sensors, and size of the tested beam, the tested beam is separated into 12 segments (E1, E2, ..., E12); each has a length of $0.5 \mathrm{~m}$ (Figure 19). The dividing points are used as excitation points; thus, there are 13 excitation points $(\mathrm{H} 1, \mathrm{H} 2, \ldots, \mathrm{H} 13)$. All these excitation points are shown in Figure 19. The damage is introduced to E3 by cutting holes on its top surface (Figure 18(b)).

The test procedure is run 24 times. As listed in

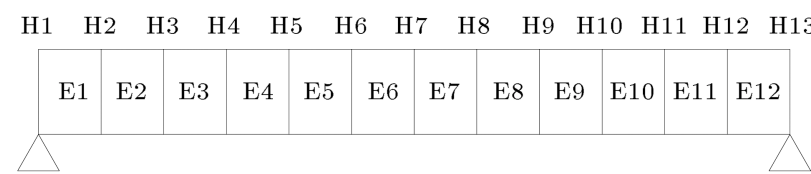

Figure 19. Segments of all the tested beams. 
Table 2. Hammer force records.

\begin{tabular}{cccc}
\hline \multirow{2}{*}{$\begin{array}{c}\text { Test } \\
\text { number }\end{array}$} & $\begin{array}{c}\text { Excited } \\
\text { point }\end{array}$ & $\begin{array}{c}\text { Without } \\
\text { damage }\end{array}$ & $\begin{array}{c}\text { With } \\
\text { damage }\end{array}$ \\
\cline { 3 - 4 } & & Hammer & force $(\mathbf{N})$ \\
\hline 1 & & 3622.82 & 2184.24 \\
2 & H3 & 2615.97 & 2212.82 \\
3 & & 1907.02 & 2135.17 \\
& & & \\
4 & & 2480.76 & 1537.82 \\
5 & H5 & 2425.00 & 2093.90 \\
6 & & 2318.37 & 1883.47 \\
7 & & & \\
8 & H8 & 2402.75 & 1883.47 \\
9 & & 1768.33 & 1781.16 \\
10 & & & \\
11 & H9 & 1506.53 & 1871.52 \\
12 & & 1961.62 & 1674.86 \\
\hline
\end{tabular}

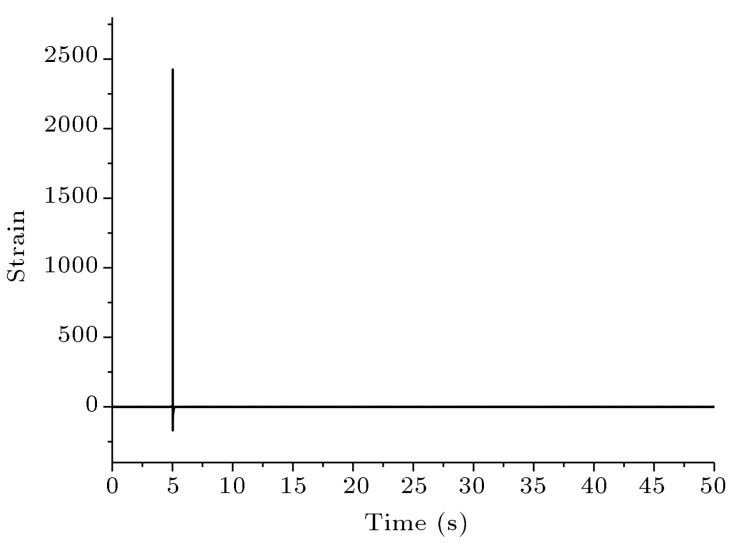

Figure 20. Hammer force time history (Test 5).

Table 2, four excitation points are selected. Six tests are recorded for each excitation point, three for the intact status, and three for damaged status.

In order to match the damage index maps, all strain signals are normalized by setting the hammer force to 1 . Table 2 shows the hammer force record of all these tests. Recorded data of Test 5 are shown in Figures 20 and 21. Figure 20 shows the hammer force time history of Test 5, which is quite clear. As can be seen in Figure 21, the strain signal is contaminated badly, with an error of about $10 \%$ of the largest strain. Figure 22 shows the instantaneous strain of both damaged and intact beams, and there is no signature that stands for the damage. Thus, the proposed damage identification method is utilized.

\subsection{Damage identification based on the data acquired by the experimental steel beam}

In this section, the data acquired during these experiments are applied to the proposed damage identifica-

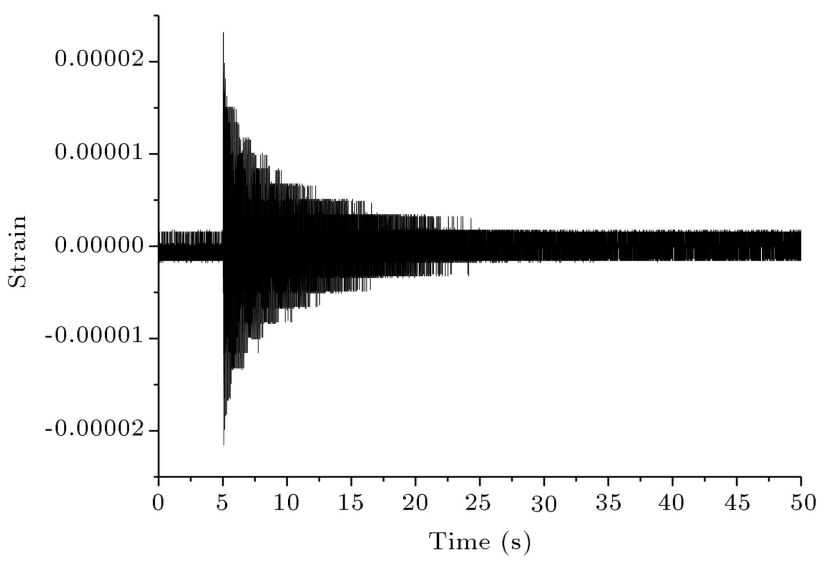

(a)

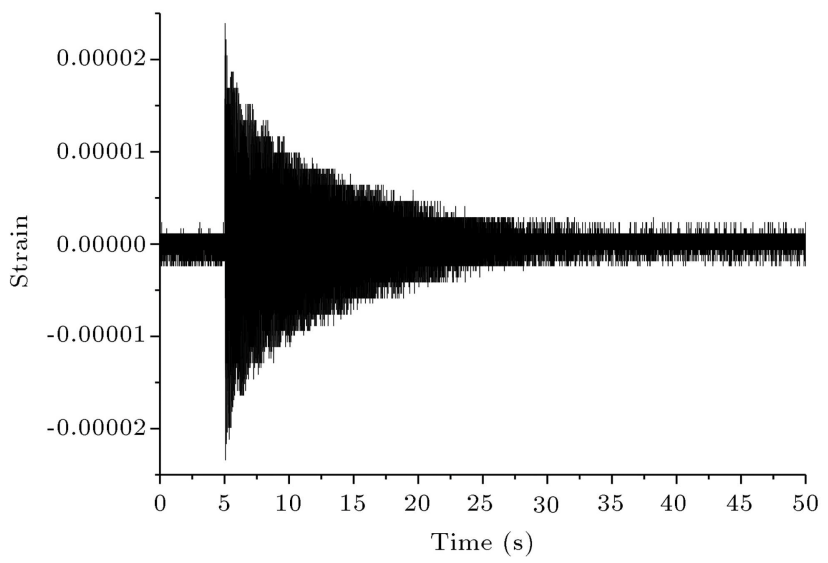

(b)

Figure 21. Strain time history of Test Number 5, intact: (a) Element 3, and (b) element 5.

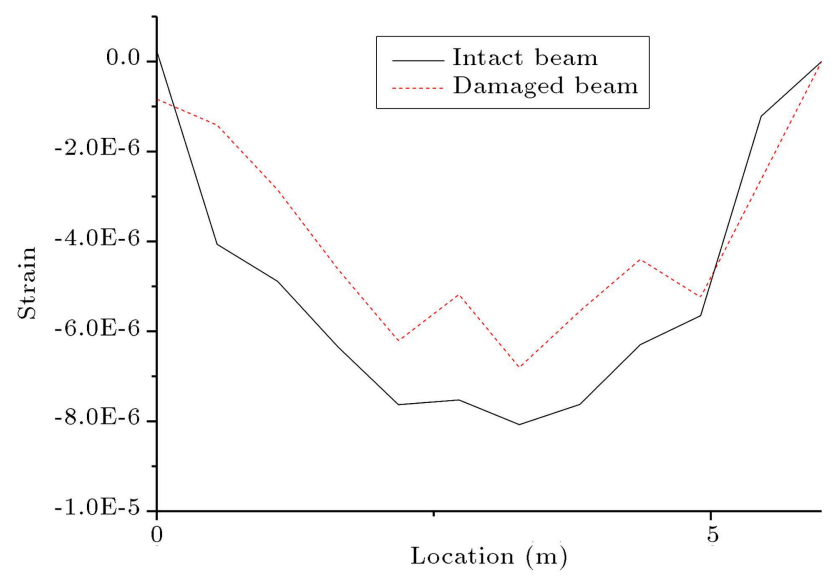

Figure 22. Instantaneous strain at time of $10 \mathrm{~s}$ (Test 5).

tion scheme. Figure 22 indicates that the instantaneous strain distributions cannot show the damage location, while instantaneous strain distribution shows the damage location by slight disturbance on the curvature (Figures (7) and (9)). This phenomenon induces noise effects, as clearly shown in Figure 21.

A damage index map is presented for a simply supported beam (Figure 14(a)). However, the excita- 


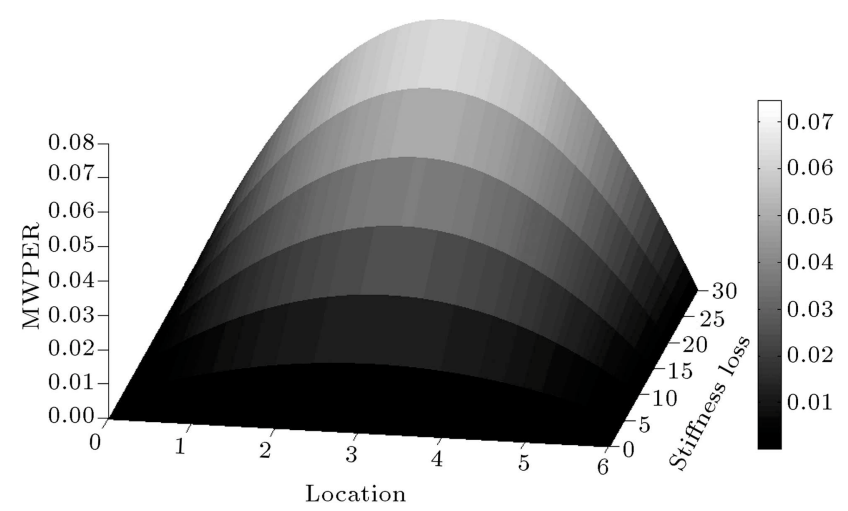

Figure 23. Damage index map for the tested beam.

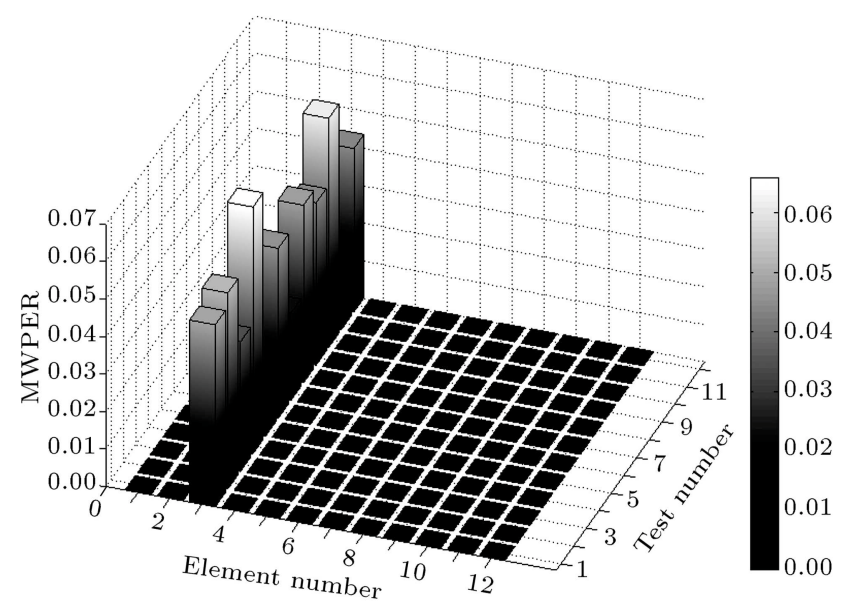

Figure 24. Damage identification result of all 12 tests.

tion conditions are different; thus, a new damage index map is calculated and presented in Figure 23.

As can be seen, all experiments' data can be utilized to identify damage locations (Figure 24). The damage is shown clearly by peaks on element 3 . However, the MPWER value of each damage identification is different from another. This situation is hardened by noise effect since the noise cannot be denoised completely.

The next step of damage identification is to identify the damage severity. The damage is found on element 3, namely location $1.5 \mathrm{~m}$. Although the MWPER values of different tests are different, they are considered to have the same importance to the damage identification result. Thus, the mean value of these 12 MWPER values is used for severity identification, which is 0.0407 . Now, two coordinates of the damaged point on the damage index map are determined. Figure 25 shows the MWPER function in position $1.5 \mathrm{~m}$. As can be seen in Figure 25, the stiffness loss level is $10 \%$, while the accurate damage level is $6.6 \%$. Although there is a large difference between the identified stiffness loss and the accurate damage level, the noise effect in measured strain signals is relatively high; this result is acceptable.

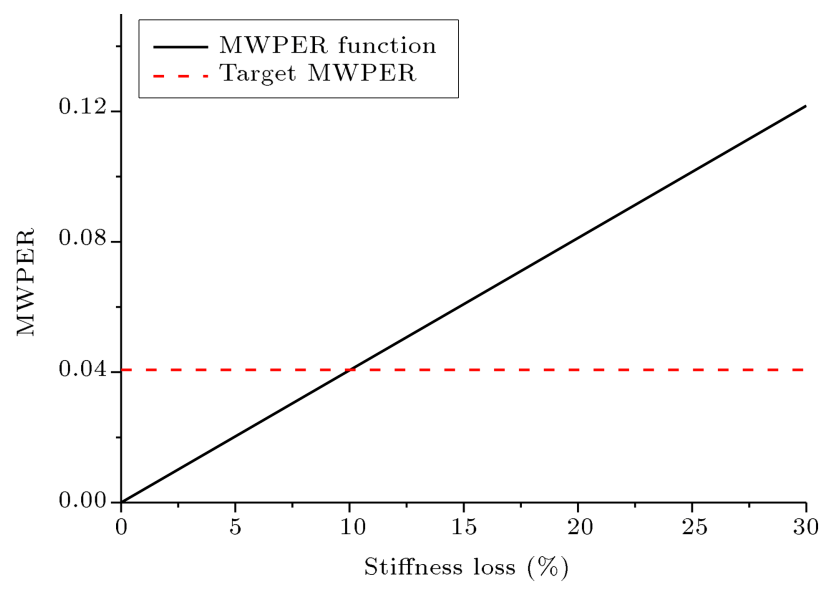

Figure 25. MWPER function in position $1.5 \mathrm{~m}$.

\section{Concluding remarks}

In this paper, MWPER was proposed for steel bridge damage identification based on long-gauge fiber optic strain sensing. With both numerical and experimental validations, the proposed method proved to be suitable for the health monitoring of the entire structure. Moreover, the proposed method was robust to noise. As presented, the damage location could be identified under a noise level of up to $30 \%$. Furthermore, it was clearly shown that the proposed scheme could be applied to identify multiple damage locations. It was also illustrated that the running car excitation was better than hammer excitation for the proposed damage identification method.

Based on the nearly linear relationship between MWPER and the stiffness loss and the relationship between MWPER and the location, three damage index maps for damage identification were established for simply supported beam, fixed end beam, and the left front column of the single layer frame. By utilizing these two maps, a corresponding map for single layer frame damage identification was established and tested. The test results showed that the proposed damage identification method was able to detect both location and severity of damages to steel bridges.

\section{Acknowledgment}

This research was supported by IIUSE of Southeast University by a grant provided by 1000 Program for the Recruitment of Global Experts. These supports are gratefully acknowledged.

\section{References}

1. Padgett, J.E. and Tapia, C. "Sustainability of natural hazard risk mitigation: Life cycle analysis of environmental indicators for bridge infrastructure", J. Infrastructure Systems, 19(4), pp. 395- 
408 (2013). https://doi.org/10.1061/(ASCE)IS.1943-5 $55 \mathrm{X} .0000138$.

2. He, C., Xing, J., Li, J., Qian, W., and Zhang, X. “A new structural damage identification method based on wavelet packet energy entropy of impulse response", The Open Civil Engineering Journal, 9, pp. 570-576 (2015).

3. Balageas, D., Fritzen, C.P., and Güemes, A., Structural Health Monitoring, Wiley-ISTE, London, pp. 493, ISBN: 978-1-905209-01-9 (2006).

4. Jiang, S.F., Wu, S.Y., and Dong, L.Q. "A timedomain structural damage detection method based on improved multiparticle swarm coevolution optimization algorithm", J. Mathematical Problems in Engineering, 2014, Article ID 232763, 11 pages, http://dx.doi.org/10.1155/2014/232763

5. Shi, Z.Y., Law, S.S., and Zhang, L.M. "Structural damage detection from modal strain energy change", J. Engineering Mechanics, 126(12), pp. 1216-1223 (2000).

https://doi.org/10.1061/(ASCE)0733-9399(2000)126: 12(1216).

6. Liu, Y., Fard, M.Y., and Chattopadhyay, A. "Damage assessment of CFRP composites using a timefrequency approach", J. Intelligent Material Systems and Structures, 23(4), pp. 397-413 (2012). DOI: https://doi.org/10.1177/1045389X11434171

7. Bu, J.Q., Law, S.S., and Zhu, X.Q. "Innovative bridge condition assessment from dynamic response of a passing vehicle", J. Engineering Mechanics - ASCE, 132(12), pp. 1372-1379 (2006).

https://doi.org/10.1061/(ASCE)0733-9399(2006)132: 12(1372).

8. McGetrick, P.J. and Kim, C.W. "Wavelet based damage detection approach for bridge structures utilising vehicle vibration", In Proceedings of 9th German Japanese Bridge, Symposium, GJBS09 (2012).

9. González, A., Obrien, E.J., and McGetrick, P.J. "Identification of damping in a bridge using a moving instrumented vehicle", J. Sound and Vibration, 331(18), pp. 4115-4131 (2012). https://doi.org/10.1016/j.jsv.2012.04.019.

10. Mallikarjuna Reddya, D., and Swarnamani, S. "Damage detection and identification in structures by spatial wavelet based approach", International Journal of Applied Science and Engineering, 10(1), pp. 69-87 (2012).

11. Wang, HF., Noori, M., and Zhao, Y. "A waveletbased damage identification for large crane structures", Sixth World Conference on Structural Control and Monitoring, pp.15-17 (2014). DOI: $10.13140 / 2.1 .2810 .7207$

12. Hera, A. and Hou, Z. "Application of wavelet approach for ASCE structural health monitoring benchmark studies", J. Engineering Mechanics, 130(1), pp. 96-104 (2004). https://doi.org/10.1061/(ASCE)07339399(2004)130:1(96).
13. Gökdağ, H. and Kopmaz, O. "A new damage detection approach for beam-type structures based on the combination of continuous and discrete wavelet transforms", J. Sound and Vibration, 324(3), pp. 1158-1180 (2009). https://doi.org/10.1016/j.jsv.2009.02.030.

14. Hester, D. and González, A. "A wavelet-based damage detection algorithm based on bridge acceleration response to a vehicle", J. Mechanical Systems and Signal Processing, 6(7), pp. 145-166 (2011). https://doi.org/10.1016/j.ymssp.2011.06.007

15. Zhao, Y., Noori, M., Altabey, W.A., and BeheshtiAval, S.B. "Mode shape based damage identification for a reinforced concrete beam using wavelet coefficient differences and multi-resolution analysis", J. Structural Control \& Health Monitoring, 25(1), pp. 1-41 (2017). https://doi.org/10.1002/stc.2041

16. Zhao, Y., Noori, M., and Altabey, W.A. "Damage detection for a beam under transient excitation via three different lagorithms", J. Structural Engineering and Mechanics, 64(6), pp. 803-817 (2017).

DOI: https://doi.org/10.12989/sem.2017.64.6.803.

17. "Identification of hysterically degrading structures using the Bouc-Wen-Baber-Noori (BWBN) model", J.F. Silva Gomes and S.A. Meguid, Editors, Proceedings IRF2018: 6th International Conference IntegrityReliability-Failure, Lisbon/Portugal, 22-26 July 2018, Publ. INEGI/FEUP, ISBN: 978-989-20-8313-1 (2018).

18. Reda Taha, M.M., Noureldin, A., Lucero, J.L., and Baca, T.J. "Wavelet transform for structural health monitoring: a compendium of uses and features", $J$. Structural Health Monitoring, 5(3), pp. 267-295 (2006). DOI: https://doi.org/10.1177/1475921706067741.

19. Nguyen, K.V. and Tran, H.T. "Multi-cracks detection of a beam-like structure based on the on-vehicle vibration signal and wavelet analysis", J. Sound and Vibration, 329(21), pp. 4455-4465 (2010). https://doi.org/10.1016/j.jsv.2010.05.005.

20. Lee, S.G., Yun, G.J., and Shang, S. "Reference-free damage detection for truss bridge structures by continuous relative wavelet entropy method", J. Struct. Health Monit, 145(2), pp. 28-45 (2014). DOI: https://doi.org/10.1177/1475921714522845.

21. Li, S. and Wu, Z. "Characterization of long-gauge fiber optic sensors for structural identification, smart structures and materials", International Society for Optics and Photonics, SPIE 5765, pp. 564-575 (2005). DOI: $10.1117 / 12.606367$

22. Horiguchi, T., Kurashima, T., and Tateda, M. "Tensile strain dependence of Brillouin frequency shift in silica optical fibers", Photonics Technology Letters, IEEE, 1(5), pp. 107-108 (1989). DOI: $10.1109 / 68.34756$.

23. Suzhen, L. and Zhishen, W. "Structural health monitoring strategy based on distributed fiber optic sensing", J. Struct. Health Monit, 6(2), pp. 133-143 (2007). DOI: https://doi.org/10.1177/1475921706072078. 
24. Dakin, J.P., Distributed Optical Fiber Sensor, International Society for Optics and Photonics, SPIE 1797, pp. 76-108 (1993).

25. Lau, K.T., Yuan, L., and Zhou, L.M. "Strain monitoring in FRP laminates and concrete beams using FBG sensors", J. Composite Structures, 51(1), pp. 920 (2001).

https://doi.org/10.1016/S0263-8223(00)00094-5.

26. Schulz, W.L., Conte, J.P., and Udd, E. "Real-time damage assessment of civil structures using fiber grating sensors and modal analysis", 9th Annual International Symposium on Smart Structures and Materials. International Society for Optics and Photonics, SPIE 4696, pp. 228-237 (2002).

27. Wei-Xin, R. and Sun, Z. "Structural damage identification by using wavelet entropy", J. Engineering Structures, 30(10), pp. 2840-2849 (2008).

https://doi.org/10.1016/j.engstruct.2008.03.013.

28. Ovanesova, A.V. and Suarez, L.E. "Applications of wavelet transforms to damage detection in frame structures", J. Engineering Structures, 26(1), pp. 39-49 (2004).

https://doi.org/10.1016/j.engstruct.2003.08.009.

29. Yongchao, Y. and Nagarajaiah, S. "Blind identification of damage in time-varying system using independent component analysis with wavelet transform", J. Mechanical Systems and Signal Processing, 27, pp. 3-20 (2012).

https://doi.org/10.1016/j.ymssp.2012.08.029.

30. Jian-Gang, H., Ren, W., and Sun, Z. "Wavelet packet based damage identification of beam structures", International Journal of Solids and Structures, 42(26), pp. 6610-6627 (2005).

https://doi.org/10.1016/j.ijsolstr.2005.04.031.

31. Yen, G.G. and Lin, K.C. "Wavelet packet feature extraction for vibration monitoring", J. Industrial Electronics, IEEE Transactions on, 47(3), pp. 650-667 (2000).

DOI: $10.1109 / 41.847906$.

32. Sun, Z., Chang, C.C. "Structural damage assessment based on wavelet packet transform", J. Structural Engineering, 128(10), pp. 1354-1361 (2002). https://doi.org/10.1061/(ASCE)0733-9445(2002)128: 10(1354).

33. Han, J.G., Ren, W.X., and Sun, Z.S. "Wavelet packet based damage identification of beam structures", Int. J. Solids and Structures, 42(26), pp. 6610-6627 (2005). https://doi.org/10.1016/j.ijsolstr.2005.04.031.

34. Maosen, C. and Pizhong, Q. "Integrated wavelet transform and its application to vibration mode shapes for the damage detection of beam-type structures", $J$. Smart Materials and Structures, IOP, 17(5), pp. 222232 (2008).

https://doi.org/10.1088/0964-1726/17/5/055014.

\section{Biographies}

Mohammad Noori is a Professor of Mechanical Engineering at California Polytechnic State University, San Luis Obispo, California, USA. He is also a Distinguished Visiting National Chair Professor of 1000 Talent Program at the International Institute for Urban Systems Engineering, Southeast University, China. Prior to Cal Poly, Professor Noori served as a Higgins Professor and the Head of Mechanical Engineering at Worcester Polytechnic Institute, as an RJ Reynolds Professor and the Head of Mechanical and Aerospace Engineering at NC State University, and as a co-founder and Liasion Professor of National Institue of Aeroscape. He has served as the Chair of the ASME National Committee of Mechancial Engineering Department Heads, as a member of numerous US-Japan cooperative research programs, sponsored by NSF, and has received the Japan Society for the Promotion of Science Fellowship. He has served as a Program Director at NSF and has held leading positions in numerous national and international scientific organizations and professional societies. Noori has carried out original research work in nonlinear random vibrations and hysteretic systems in multi-functional structures; for the past 15 years, his research has focused on the utilization of Ai-based schemes for structural health monitoring. He is an ASME Fellow, has published over 200 refereed papers, and serves as an executive editor, an associate editor, and an editorial board member of several journals, as a technical advisor and board member of numerous scientific organizations and industries, and has delivered over 120 keynote and invited talks. He is an elected member of several professional honor societies.

Haifegn Wang received Master's degree at Southeast University under the supervision of Professor Noori, a Distinguished Visiting National Chair Professor of China 1000 Talent Program at the International Institute for Urban Systems Engineering, Southeast University, China. He is currently a PhD student at the state University of New York in Buffalo, USA.

Wael A. Altabey is an Assistant Professor of Mechanical Engineering, Faculty of Engineering, Alexandria University, Alexandria, Egypt. He is currently an Associate Researcher and a visiting scholar at the International Institute for Urban Systems Engineering, in Southeast University and at Nanjing Zhixing Information Technology Co., Ltd., Nanjing, China. He was awarded a postdoctoral research certificate in 2018 after completing a post-doctoral research fellowship for two years, under the supervision of Professor Noori, in SHM and damage detection from Southeast University, Nanjing, China. Dr. Altabey received his PhD in 2015 in the area of fatigue of composite structures and his 
MSc, 2009, in dynamic systems and his BSc, 2004, in Mechanical Engineering, from Mechanical Engineering Department, Faculty of Engineering, Alexandria University. Currently, Dr. Altabey is carrying out several projects related to the application of artificial intelligence methods for structural health monitoring.
Ahmed I.H. Silik is currently a PhD student at the International Institute for Urban Systems Engineering, Southeast University, Nanjing, China. His research work involves the utilization of wavelet and artificial neural network for the damage detection of reinforced concrete structures. 